\title{
MODELING THE EFFECT OF CONTACT AND SEEPAGE FORCES AT EQUILIBRIUM ON THE FAILURE OF WATER BOREHOLE
}

\author{
K. C. Onyelowe ${ }^{1^{*}}$ and F. 0. Okafor ${ }^{2}$ \\ 1 Dept. of Civil Engineering, Michael OKPARA Univ. of Agriculture, Umudike, Umuahia, ABia State. NigERIA \\ 2 DEPARTMENT OF CiVIL ENGINEERING, UNIVERSITY OF NIGERIA, NSUKKA. ENUGU STATE. NIGERIA \\ E-mail addresses: ${ }^{1}$ konyelowe@mouau.edu.ng, 2 fidelis.okafor@unn.edu.ng
}

\section{ABSTRACT}

There have been records of failures and quicksand conditions in boreholes in recent times impeding the performance and operation of boreholes which may have resulted from various factors ranging from construction problems, drilling inaccuracies, fitting and installation problems, some chemical effects within the aquifer medium etc, but it has been ignored that a factor of great benefit to the operation of water boreholes; seepage force could get to a considerable which becomes unsafe for the well operation thereby causing dislodgement of sand particles and sandstones resulting boiling. This research work has investigated the contribution of contact force and seepage force to the failure of boreholes. This necessitated the use of combined finite-discrete element method to generate model expressions from contact and seepage forces considered to be the major forces contributing to the flow of fluid through soil mass and boiling or quicksand effect results when seepage force becomes more in effect under critical hydraulic gradient and / or critical hydraulic head. A mathematical/laboratory model was used and an expression for calculating the critical hydraulic head causing critical seepage deduced as $\boldsymbol{h}_{(x)}$ $=0.000524 r^{2}\left[X_{5}\left(5-2 S F_{x_{2}}\right)\right]$ and the equilibrium model has deduced an expression for the safe hydraulic head during well pumping as $\boldsymbol{H}_{(x)}=0.0065814 . r^{4} X_{5} \aleph_{x_{2}}$. These have been verified using a laboratory investigation; borehole prototype well failure test. It has been established that there is strong agreement between model result and the laboratory study result from the correlation analysis conducted which has shown correlations of 1.00975 and 0.989879999701 for the critical state condition and equilibrium state condition respectively. For purposes of future calculations, borehole performance monitoring and designs, the standard critical hydraulic head of the system from Table 3 and Fig.7 is 2.92E-8 which has the strongest agreement with 2.59E-8 of the laboratory study with a deviation of 3.3E-9. The deduced models can be used to design and monitor the performance of boreholes. For safe pumping and corresponding yield in the bore hole system, inter-granular force between granular particles should equal the seepage force and this is achieved by ensuring that the deduced model expression is used to determine the safe hydraulic head. Finally, irrespective of the fact that an increase in hydraulic head increases discharge, the system should be operated at a head safe for the performance of the well and as long as the model hydraulic head expression deduced is used under the above conditions, safe pumping can be achieved at any voltage between 150volts and 240volts.

Keywords: Equilibrium; contact force; seepage force; modelling; water borehole; failure.

\section{INTRODUCTION}

Identification and establishment contact and seepage forces as factors that contribute to the failure of boreholes are the main targets of this research work. The medium under study is a solid-liquid medium with the liquid migrating through the voids of the solid (granular soil) to where it is pumped for use. During this process, fluid moves from point to point introducing forces acting both on the fluid and the granular material causing dislodgment and displacement of the particles which are collected at the walls of the well casing. These particles also block the well casing perforations or screens making the well casing inefficient to transmit the collected fluid into the well for pumping [1]. Two critical factors have been identified for study in the present research work 
as those that cause the failure of water boreholes operation thus;

1. Interaction force between the soil particles (restoring force).

2. The force causing dislodgment of particles

The above factors are to be extensively studied to arrive at an equilibrium model and solution to the problem under study. Nigeria has a total land mass of 932,768Sq.Km falling between latitude 401 and $13^{09}{ }^{1} \mathrm{~N}$ and longitudes $2^{0} 2^{1}$ and $14^{0} 3^{1} \mathrm{~W}$ and a population, currently of about 120 million people [2]. The total replenishable water resource in Nigeria is estimated at 319 billion cubic meters, while the ground water component is estimated at 52 billion cubic meters. Water shortages are acute in some major centers and in numerous rural communities due to a variety of factors including variation in climatic conditions, drought increasing demands, distribution system losses and breakdown of works and facilities [2]. Ground water is the water stored in an aquifer in pore spaces or fractures in rocks or sediments. Groundwater is generally a readily available source of water throughout populated Africa but the construction costs for sustainable supplies are high. The reason why groundwater is preferred to surface water includes:

- Its relative low costs compared to surface water

- Availability in most areas

- Potable without treatment

- Employs low cost technologies

- The frequent drought problems enforce the use of groundwater source as many small intermittent rivers and streams dry out during the dry seasons.

\subsection{Groundwater development in Nigeria}

The establishment of the Nigerian geological Survey in 1919 has as one of its major objectives to search for groundwater in the semiarid areas of the former northern Nigeria. These activities by the Nigerian Geological Survey culminated in the commencement in 1928 of systematic investigations of towns and villages for the digging of hand dug wells. In 1938, a water drilling section of the geological survey was setup and by 1947, the engineering aspects of the water supply section were handed over to the Public Works Department, which is the forerunner of the present Ministry of Works while the Geological Survey maintained the Exploration functions. The aim of studying borehole failures is to identify the factors responsible for borehole engineering solutions.
According to [2], the most plausible causes of these borehole failures can be attributed to

(i) Design and construction

(ii) Groundwater potential/ hydro geological consideration and

(iii) Operational and maintenance failures.

With the foregoing, [2] has failed to recognize the purely engineering factors that could cause the failure of boreholes and this has stimulated the present research work to establish seepage and contact forces as the two major opposing physical factors that fall within the scope of the present work for study.

\subsection{The Combined Finite-Distinct Element Method}

The combined FDEM is aimed at problems involving transient dynamics of systems comprising a large number of deformable bodies that interact with each other, and that may in general fracture and fragment, thus increasing the total number of discrete (distinct) elements even further. Each individual distinct element is of a general shape and size, and is modeled by a single distinct element. Each distinct element is discretized into finite elements to analyze deformability, fracture and fragmentation. A typical combined FDEM system comprises a few thousand to a few million separate interacting solids, each associated with separated finite element meshes [3; 4; 5]. In this work, one of the key issues in the development of the combined FDEM is the treatment of contact between the elements, fluid flow through the voids between the elements and the displacement of the elements. The only numerical tool currently available to a scientist or engineer that can properly take systems comprising millions of deformable distinct elements that simultaneously fracture and fragment under both fluid and solid phase is the combined FDEM. The combined FDEM merges finite element tools and techniques with distinct element algorithms $[5 ; 6 ; 7 ; 8]$. Finite element based analysis of continua is merged with distinct element-based transient hydrodynamics, contact detection and contact interaction solutions. Thus, transient dynamic analysis of systems comprising a large number from a few thousands to more than a million of deformable bodies which interact with each other and in through seepage process can break fracture or fragment, becomes possible [3].

\section{METHODOLOGY AND FORMULATION}

Contact force (inter-granular force) and seepage force are two fundamental physical phenomena under study 
in the present work because of their pronounced effect on the failure of the walls of water boreholes. They are two opposing forces i.e. disturbing and restoring forces and therefore deserve our keen attention and study. The basic principle involved in the formulation is the combined FDEM because of the continuum and discontinuum nature of the studied region. From the foregoing, the problem of contact force (intergranular force) existing within the region of the soil mass or volume is a discontinuum problem, and therefore employs discrete element method in the formulation of the matrix contact force equation where every particle that make up the soil mass is considered a discrete element. Similarly, the problem of volume force or seepage force is a continuum problem and employs the finite element method in its formulation

\subsection{Contact Force Model}

Contact interaction between neigbouring distinct elements occurs through solid surfaces as illustrated in Figure 1 which are generally irregular and as a consequence, the contact pressure between two solids is acutally transferred through a set of points, and with increasing normal stresses, surfaces only touch at a few points. With increasing normal stresses, elastic and plastic deformation of individual surface asperities occur, resulting in an increase in the real contact area [3].

Problems of contact interaction in the context of the combined FDEM are even more important, due to the fact that in this method, the problem of contact interaction and handling of conext also defines the constitutive behaviour of the system, because of the presence of large numbers of separate bodies. Thus, algorithms employed must pay special attention to contact kinematic in terms of the realistic distribution of contact forces, energy balance and robustness [3]. The present research on contact interaction algorithm makes use of finite element discretizations of discrete elements, and combines this with the so-called potential (pressure/stress) contact force concept. This algorithm assume discretization of individual discrete elements into finite elements, thus imposing no additional database requirements in handling the geometry of individual discrete elements. They also yield realistic distribution of contact for use over finite contact area resulting from the overlap of discrete elements that are in contact.

The distributed contact force is adopted for two discrete elements in contact, shown in Figure 1, one of which is denoted as the contactor $\mathrm{C}$ and the other as the target, $\mathrm{t}$. When in contact, the contactor and target discrete elements overlap each other over area S, bounded by boundary (Figure 2).

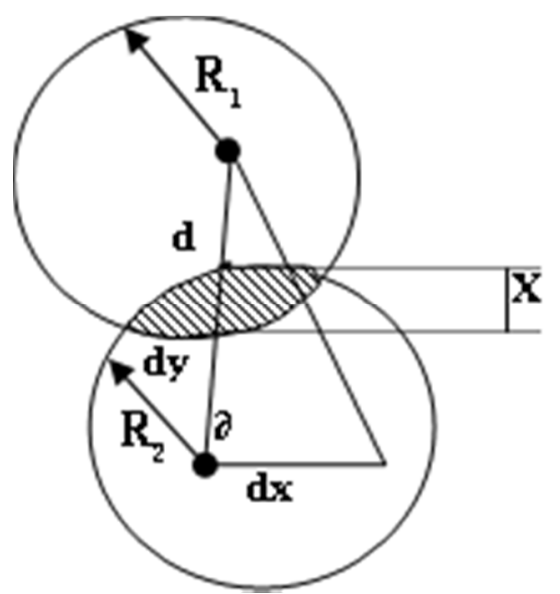

Figure 1: Particle contact geometry

It is assumed that penetration of any elementary area $\mathrm{dA}$ of the contactor into the target results in an infintesimal contact force, given by

$\mathrm{dF}=\left[\operatorname{grad} . \psi_{\mathrm{c}}(\mathrm{Pc})+\operatorname{grad} . \psi_{\mathrm{t}}(\mathrm{Pt})\right] \mathrm{dA}$

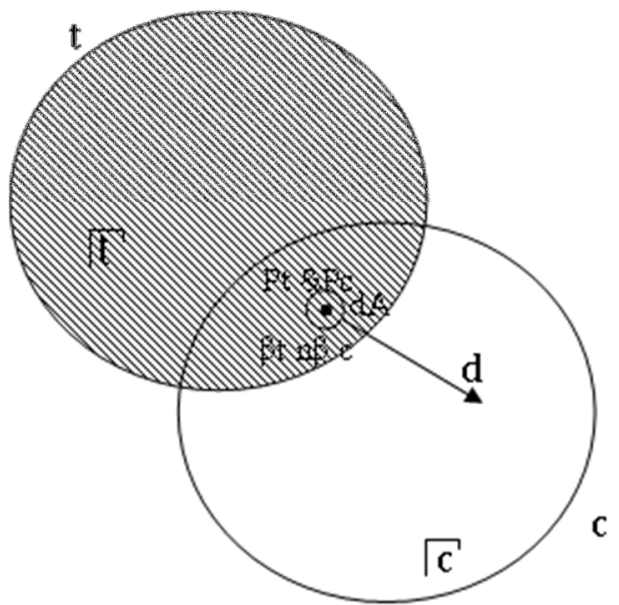

Figure 2: Contact force due to an infinitesimal overlap around points $P c$ and $P t$

In (1), $d F$ is theInfinitesimal contact force, $d A$ is the Infinitesimal area, $\psi(p)$ is the Potential function, $\sigma_{c}$, $\sigma_{\mathrm{t}}$, and is the Contactor and target stresses

Equation 1 can be written as

$\mathrm{dF}=\mathrm{dFt}+\mathrm{dFc}$

Where

$\mathrm{dFc}=\operatorname{grad} \psi_{\mathrm{t}}(\mathrm{Pt}) \mathrm{dAc}$,

$\mathrm{dAc}=\mathrm{dA}$

$\mathrm{dFt}=\operatorname{grad} \psi_{\mathrm{c}}(\mathrm{Pc}) \mathrm{dAt}, \quad(5)$

$\mathrm{dAt}=\mathrm{dA}$

Considering a third discrete element known as supporter discrete element $\mathrm{S}$ and its effects on the contact force, Equation 2 will become, 
$\left[\begin{array}{l}F_{c} \\ F_{t} \\ F_{s}\end{array}\right]=\left[\begin{array}{ccccc}\sigma_{\mathrm{c}_{1}} & \sigma_{\mathrm{c}_{2}} & \ldots \ldots \ldots \ldots \sigma_{\mathrm{c}_{\mathrm{n}}} \\ \sigma_{\mathrm{t}_{1}} & \sigma_{\mathrm{t}_{2}} & \ldots \ldots \ldots \ldots \sigma_{\mathrm{t}_{\mathrm{n}}} \\ \sigma_{\mathrm{s}_{1}} & \sigma_{\mathrm{s}_{2}} & \ldots \ldots \ldots \ldots \sigma_{\mathrm{s}_{\mathrm{n}}}\end{array}\right] \quad\left[\begin{array}{c}A_{1} \\ A_{2} \\ \vdots \\ A_{n}\end{array}\right]$

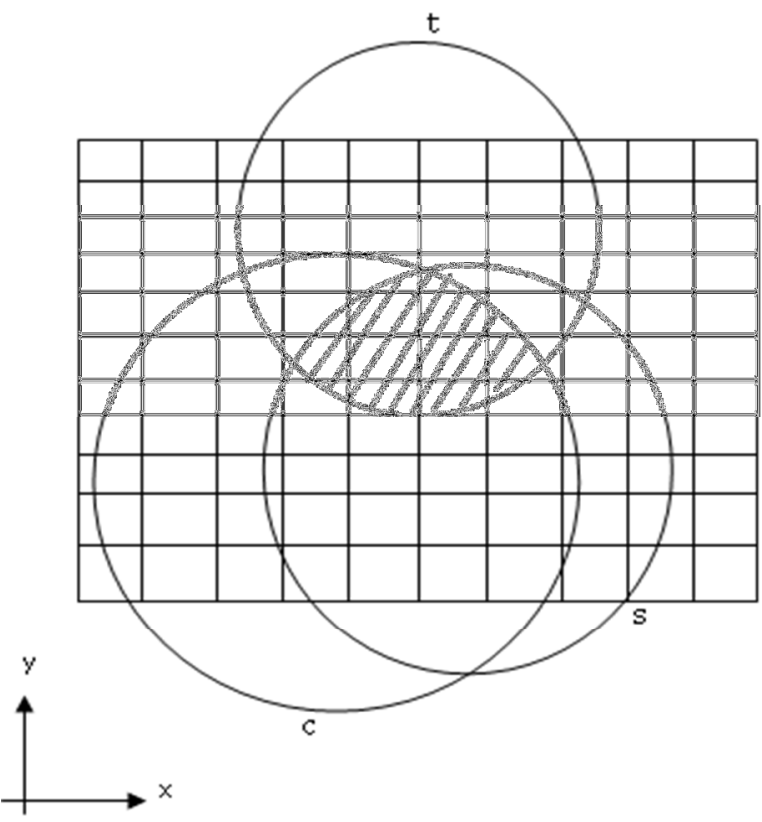

Figure 3: Discretisation of contactor, target and support discrete elements contact zone to finite elements

\subsection{Seepage Force Model}

Soils are premeable to fluids (water) because the voids between soil particles are interconnected. The degree of permeability is characterized by the permeability coefficient $\mathrm{K}$, also referred to as hydraulic conductivity. The basic concepts of seepage and flow through granualr soil materials viz fluid velocity, seepage quantity, discharge velocity, hydraulic gradient etc. obey Darcy's law thus

$\mathrm{q}=\mathrm{KiA}$

In (8), $Q$ is the discharge in $\mathrm{m}^{3} / \mathrm{s}, \mathrm{K}$ is the hydraulic conductivity or permeability constant, I is the hydraulic gradient, and A is the cross section area of flow region.

Three discrete particles; target, contactor and support particles and the fluid flow through the contact zone were considered as in Fig. 3;

In strict agreement with [13]; seepage force (Fig.5) as a volume force is given by the expression [14],

$S F=i . \gamma_{W}$

Where I is the hydraulic gradient and $\gamma_{w}$ is the unit weight of water $\mathrm{KN} / \mathrm{m}^{3}$

Consider the elemental area under study, the elemental hydraulic head $\mathrm{dH}$ that causes flow of water in the soil mass or volume is given as
$\mathrm{dH}=\mathrm{SF} \cdot \mathrm{dx} \cdot \mathrm{Yw}^{-1}$

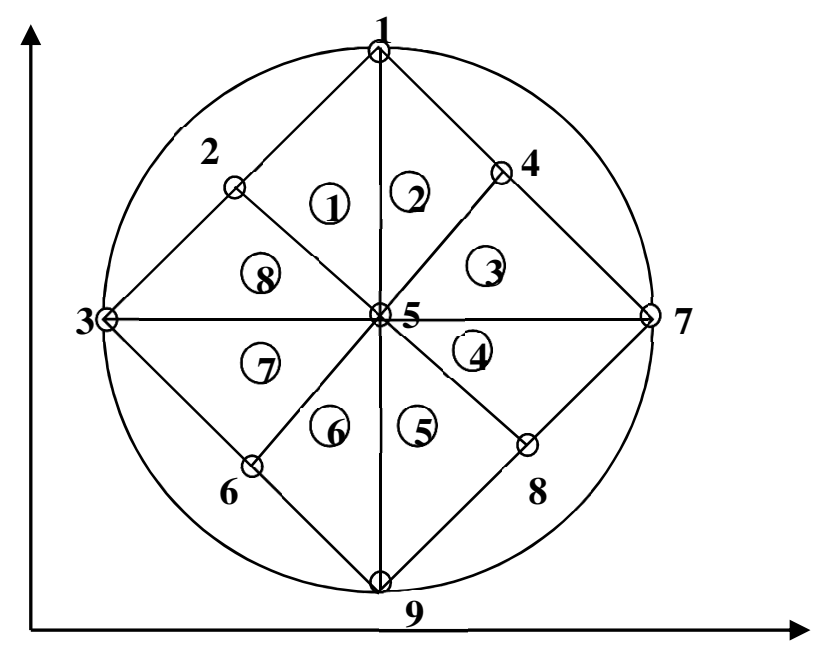

Figure 4: Elements and nodal points of the contact zone

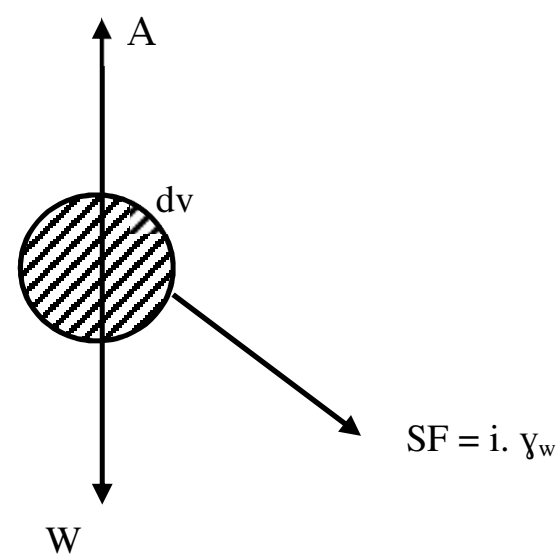

Figure 5: Soil volume subjected to three force components

The matrix formation of Equation 10 above will give;

$\left[\begin{array}{c}H_{x} \\ H_{y} \\ H_{z}\end{array}\right]=\frac{1}{\gamma_{w}}\left[\begin{array}{lllll}S F_{x 1} & S F_{x 2} & S F_{x 3} & \cdots & S F_{x n} \\ S F_{y 1} & S F_{y 2} & S F_{y 3} & \cdots & S F_{y n} \\ S F_{z 1} & S F_{z 2} & S F_{z 3} & \cdots & S F_{z n}\end{array}\right]\left[\begin{array}{c}X_{1} \\ X_{2} \\ X_{3} \\ \vdots \\ X_{n}\end{array}\right]$

According to [15] there is need to choose a shape function from the descretized contact zone in Fig.4 for the nodal fluid potential;

$$
\begin{gathered}
H=H_{1}\left(1-\frac{X}{L}\right)+H_{2}\left(\frac{X}{L}\right) \\
H=[N]\left\{H_{n}\right\}
\end{gathered}
$$

And the element formulation is given as;

$$
\frac{A . S F}{L}\left[\begin{array}{ccc}
1 & -1 & 1 \\
-1 & 1 & -1 \\
1 & -1 & 1
\end{array}\right]\left\{\begin{array}{l}
X_{1} \\
X_{2} \\
X_{3}
\end{array}\right\}=f_{(x)} \frac{H}{2}\left\{\begin{array}{l}
1 \\
1 \\
1
\end{array}\right\}
$$

Or,

$$
[S F]\left\{X_{n}\right\}=\{H\}
$$

Equation 13 is to be applied to all the elements of the mesh as shown in Fig. 8 to develop element equation for each of the elements of the zone. 
Element 1; nodes 1, 2, and 5

$$
\frac{A}{\gamma_{w} L}\left[\begin{array}{ccc}
-S F_{x 1} & S F_{x 2} & -S F_{x 5} \\
S F_{y 1} & -S F_{y 2} & S F_{y 5} \\
-S F_{z 1} & S F_{z 2} & -S F_{z 5}
\end{array}\right]\left[\begin{array}{l}
X_{1} \\
X_{2} \\
X_{5}
\end{array}\right]
$$

Element 2; nodes 1,4 , and 5

$$
\frac{A}{\gamma_{w} L}\left[\begin{array}{ccc}
-S F_{x 1} & S F_{x 4} & -S F_{x 5} \\
S F_{y 1} & -S F_{y 4} & S F_{y 5} \\
-S F_{z 1} & S F_{z 4} & -S F_{z 5}
\end{array}\right]\left[\begin{array}{l}
X_{1} \\
X_{4} \\
X_{5}
\end{array}\right]=f_{(x)} \frac{H}{2}\left[\begin{array}{c}
1+1 \\
1+1 \\
1+1
\end{array}\right]
$$

Element 3; nodes 4, 5, and 7,

$$
\frac{A}{\gamma_{w} L}\left[\begin{array}{ccc}
-S F_{x 4} & S F_{x 5} & -S F_{x 7} \\
S F_{y 4} & -S F_{y 5} & S F_{y 7} \\
-S F_{z 4} & S F_{z 5} & -S F_{z 7}
\end{array}\right]\left[\begin{array}{l}
X_{4} \\
X_{5} \\
X_{7}
\end{array}\right]=f_{(x)} \frac{H}{2}\left[\begin{array}{l}
1+1 \\
1+1 \\
1+1
\end{array}\right]
$$

Element 4; nodes 5, 7, and 8,

$$
\frac{A}{\gamma_{w} L}\left[\begin{array}{ccc}
-S F_{x 5} & S F_{x 7} & -S F_{x 8} \\
S F_{y 5} & -S F_{y 7} & S F_{y 8} \\
-S F_{z 5} & S F_{z 7} & -S F_{z 8}
\end{array}\right]\left[\begin{array}{c}
X_{5} \\
X_{7} \\
X_{8}
\end{array}\right]=f_{(x)} \frac{H}{2}\left[\begin{array}{c}
1+1 \\
1+1 \\
1+1
\end{array}\right]
$$

Element 5; nodes 5, 8, and 9,

$$
\frac{A}{\gamma_{w} L}\left[\begin{array}{ccc}
-S F_{x 5} & S F_{x 8} & -S F_{x 9} \\
S F_{y 5} & -S F_{y 8} & S F_{y 9} \\
-S F_{z 5} & S F_{z 8} & -S F_{z 9}
\end{array}\right]\left[\begin{array}{l}
X_{5} \\
X_{8} \\
X_{9}
\end{array}\right]=f_{(x)} \frac{H}{2}\left[\begin{array}{c}
1+1 \\
1+1 \\
1+1
\end{array}\right]
$$

Element 6; nodes 5, 6, and 9,

$$
\begin{array}{r}
\frac{A}{\gamma_{w} L}\left[\begin{array}{ccc}
-S F_{x 5} & S F_{x 6} & -S F_{x 9} \\
S F_{y 5} & -S F_{y 6} & S F_{y 9} \\
-S F_{z 5} & S F_{z 6} & -S F_{z 9}
\end{array}\right]\left[\begin{array}{l}
X_{5} \\
X_{6} \\
X_{9}
\end{array}\right] \\
=f_{(x)} \frac{H}{2}\left[\begin{array}{l}
1+1 \\
1+1 \\
1+1
\end{array}\right]
\end{array}
$$

Element 7; nodes 3, 5, and 6,

$$
\begin{array}{r}
\frac{A}{\gamma_{w} L}\left[\begin{array}{ccc}
-S F_{x 3} & S F_{x 5} & -S F_{x 6} \\
S F_{y 3} & -S F_{y 5} & S F_{y 6} \\
-S F_{z 3} & S F_{z 5} & -S F_{z 6}
\end{array}\right]\left[\begin{array}{l}
X_{3} \\
X_{5} \\
X_{6}
\end{array}\right] \\
=f_{(x)} \frac{H}{2}\left[\begin{array}{c}
1+1 \\
1+1 \\
1+1
\end{array}\right]
\end{array}
$$

Element 8; nodes 2, 3, and 5,

$$
\begin{array}{r}
\frac{A}{\gamma_{w} L}\left[\begin{array}{ccc}
-S F_{x 2} & S F_{x 3} & -S F_{x 5} \\
S F_{y 2} & -S F_{y 3} & S F_{y 5} \\
-S F_{z 2} & S F_{z 3} & -S F_{z 5}
\end{array}\right]\left[\begin{array}{c}
X_{2} \\
X_{3} \\
X_{5}
\end{array}\right] \\
=f_{(x)} \frac{H}{2}\left[\begin{array}{c}
1+1 \\
1+1 \\
1
\end{array}\right]
\end{array}
$$

The global matrix equation assembled from the eight element equations and substituting the following boundary conditions;

$$
\begin{gathered}
\quad{ }^{5} S F_{\text {odd }}=0 \\
S F_{5}=1 \\
0<\mathrm{SF}_{\text {even }}<1 \\
0<\mathrm{X}<\mathrm{L}
\end{gathered}
$$

Simplifying further gives (28)

$$
\left[\begin{array}{c}
X_{2}\left(-S F_{x 2}\right)+X_{5} \\
X_{2}\left(S F_{y 2}\right)-X_{5} \\
X_{2}\left(-S F_{z 2}\right)+X_{5} \\
X_{4}\left(-S F_{x 4}\right)+X_{5} \\
X_{4}\left(S F_{y 4}\right)-X_{5} \\
X_{4}\left(-S F_{z 4}\right)+X_{5} \\
X_{4}\left(S F_{x 4}\right)-X_{5} \\
X_{4}\left(-S F_{y 4}\right)+X_{5} \\
X_{4}\left(S F_{z 4}\right)-X_{5} \\
X_{5}+X_{8}\left(S F_{x 8}\right) \\
-X_{5}+X_{8}\left(-S F_{y 8}\right) \\
X_{5}+X_{8}\left(S F_{z 8}\right) \\
X_{5}+X_{8}\left(-S F_{x 8}\right) \\
-X_{5}+X_{8}\left(S F_{y 8}\right) \\
X_{5}+X_{8}\left(-S F_{z 8}\right) \\
X_{5}+X_{6}\left(-S F_{x 6}\right) \\
-X_{5}+X_{6}\left(S F_{y 6}\right) \\
X_{5}+X_{6}\left(-S F_{z 6}\right) \\
-X_{5}+X_{6}\left(S F_{x 6}\right) \\
X_{5}+X_{6}\left(-S F_{y 6}\right) \\
-X_{5}+X_{6}\left(S F_{z 6}\right) \\
X_{2}\left(S F_{x 2}\right)+X_{5} \\
X_{2}\left(-S F_{x 2}\right)-X_{5} \\
X_{2}\left(S F_{x 2}\right)+X_{5}
\end{array}\right]=\left[\begin{array}{c}
\frac{H_{1}}{2} \\
H_{2} \\
H_{5} \\
H_{1} \\
H_{4} \\
H_{5} \\
H_{4} \\
H_{5} \\
H_{7} \\
H_{5} \\
H_{7} \\
H_{8} \\
H_{5} \\
H_{8} \\
H_{9} \\
H_{5} \\
H_{6} \\
H_{9} \\
H_{3} \\
H_{5} \\
H_{6} \\
H_{2} \\
H_{3} \\
\frac{H_{5}}{2}
\end{array}\right]
$$

This implies that;

$$
\begin{aligned}
& h_{(x)}=\frac{A}{\gamma_{w} \cdot L}\left\{\left[X_{2}\left(-S F_{x 2}\right)+X_{5}\right]+\left[X_{2}\left(S F_{y 2}\right)-X_{5}\right]+\right. \\
& {\left[X_{2}\left(-S F_{z 2}\right)+X_{5}\right]+\left[X_{4}\left(-S F_{x 4}\right)+X_{5}\right]+} \\
& {\left[X_{4}\left(S F_{y 4}\right)-X_{5}\right]+\left[X_{4}\left(-S F_{z 4}\right)+X_{5}\right]+\left[X_{4}\left(S F_{x 4}\right)-\right.} \\
& \left.X_{5}\right]+\left[X_{4}\left(-S F_{y 4}\right)+X_{5}\right]+\left[X_{4}\left(S F_{z 4}\right)-X_{5}\right]+ \\
& {\left[X_{5}+X_{8}\left(S F_{x 8}\right)\right]+\left[-X_{5}+X_{8}\left(-S F_{y 8}\right)\right]+} \\
& {\left[X_{5}+X_{8}\left(S F_{z 8}\right)\right]+\left[X_{5}+X_{8}\left(-S F_{x 8}\right)\right]+} \\
& {\left[-X_{5}+X_{8}\left(S F_{y 8}\right)\right]+\left[X_{5}+X_{8}\left(-S F_{z 8}\right)\right]+} \\
& {\left[X_{5}+X_{6}\left(-S F_{x 6}\right)\right]+\left[-X_{5}+X_{6}\left(S F_{y 6}\right)\right]+} \\
& {\left[X_{5}+X_{6}\left(-S F_{z 6}\right)\right]+\left[-X_{5}+X_{6}\left(S F_{x 6}\right)\right]+} \\
& {\left[X_{5}+X_{6}\left(-S F_{y 6}\right)\right]+\left[-X_{5}+X_{6}\left(S F_{z 6}\right)\right]+\left[X_{2}\left(S F_{x 2}\right)+\right.} \\
& \left.\left.X_{5}\right]+\left[X_{2}\left(-S F_{x 2}\right)-X_{5}\right]+\left[X_{2}\left(S F_{x 2}\right)+X_{5}\right]\right\}
\end{aligned}
$$

Colleting like terms and simplifying Equation 29, we will have;

$$
\begin{aligned}
h_{(x)}=\frac{A}{\gamma_{w} L}\left[X_{5}(\right. & \left.\left.-2 S F_{x_{2}}\right)\right] \\
= & 0.000524 r^{2}\left[X_{5}\left(5-2 S F_{x_{2}}\right)\right]
\end{aligned}
$$

Where, $S F_{x_{2}}$ is the seepage force in the flow system, $=[0.1,0.2,0.3 \ldots 1.0][3]$,

$\mathrm{r}$ is the average radius of discrete particle $=$ $0.002857 \mathrm{~m}, \mathrm{X}$ is the flow distance, $\gamma_{w}$ is the unit weight of water $=1000 \mathrm{~kg} / \mathrm{m}^{3}$, L is the cross sectional length of the flow medium $=6 \mathrm{~m}$ and $\mathrm{X}_{5}$ varies between 0.6 and 6.0 .

Equation 30 fulfils the condition of critical hydraulic gradient that causes sand boiling or quick sand effect in the flow region. 


\subsection{Equilibrium Condition of Studied Region}

Under equilibrium conditions, there is fluid flow without its attendant particle dislodgement and displacement. This emplies that at this state, the disturbing force and the restoring force are equal or the algebraic sum of the fundamental forces equals zero. Thus contact force equals seepage force

That is to say that,

$\mathrm{NdA}-\mathrm{i} . \mathrm{y}_{\mathrm{w}}=0$

Where $\mathrm{N}$ is the contact stress of the region, $\mathrm{dA}$ is the elemental surface area of granualr particles, iis the hydraulic gradient and $\gamma_{w}$ is the unit weight of water

$$
\text { א.dA } \quad \text { i. } \quad \text { w }
$$

$\aleph . \mathrm{dA}$

$$
=\quad \frac{\mathrm{dH}}{\mathrm{dx}} \cdot \gamma_{\mathrm{w}}
$$

w.dA.dx

$$
=\mathrm{dH} \cdot \mathrm{\gamma}_{\mathrm{w}}
$$

$\mathrm{dH}$

$$
=\quad \frac{1}{\gamma_{w}} \cdot \aleph \cdot d A \cdot d x
$$

Where $\frac{\mathrm{dA}}{\gamma_{\mathrm{w}}}$ is a constant

$$
\mathrm{dH}=\frac{\mathrm{dA}}{\gamma_{\mathrm{w}}} \times \mathrm{N} \cdot \mathrm{dx}
$$

The stress between particles as they come in contact and are held together by contact force varies node to node and from particle to partilce in the direction of flow. However, within the three directions of flow $\mathrm{x}, \mathrm{y}$ and $z$, the head at which the borehole is to be operated to forestall failure of the soil medium by dislodgement of the particles or grains that make the soil volume or mass is calculated as,

$$
\mathrm{dH}=\mathrm{Hx}+\mathrm{Hy}+\mathrm{Hz}
$$

The stress of the domain $\mathrm{Nin}$ three directional are:

$$
\begin{aligned}
& \aleph_{\mathrm{x}}=\kappa_{\mathrm{x} 1}+\kappa_{\mathrm{x} 2}+\kappa_{\mathrm{x} 3}+\ldots \ldots \ldots . . . \kappa_{\mathrm{xn}} \\
& \aleph_{\mathrm{y}}=\kappa_{\mathrm{y} 1}+\kappa_{\mathrm{y} 2}+\kappa_{\mathrm{y} 3}+\ldots \ldots \ldots . . . \aleph_{\mathrm{yn}} \\
& \aleph_{\mathrm{z}}=\aleph_{\mathrm{z} 1}+\aleph_{\mathrm{z} 2}+\aleph_{\mathrm{z} 3}+\ldots \ldots \ldots \ldots \aleph_{\mathrm{zn}}
\end{aligned}
$$

Equation 15 becomes

$$
\mathrm{H}=\sum_{\mathrm{x}=1}^{\mathrm{n}} \aleph_{\mathrm{x}}+\sum_{\mathrm{y}=1}^{\mathrm{n}} \aleph_{\mathrm{y}}+\sum_{\mathrm{z}=1}^{\mathrm{n}} \aleph_{\mathrm{z}}\left[\int \frac{\mathrm{dA}}{\gamma_{\mathrm{w}}} \aleph^{\mathrm{n}} \cdot \mathrm{dx}\right]
$$

The matrix tranformation of Equation 18 becomes

$$
\left[\begin{array}{c}
H_{x} \\
H_{y} \\
H_{z}
\end{array}\right]=\frac{A}{\gamma_{w}}\left[\begin{array}{ccccc}
\aleph_{\mathrm{x} 1} & \aleph_{\mathrm{x} 2} & \aleph_{\mathrm{x} 3} & \ldots \ldots . & \aleph_{\mathrm{xn}} \\
\aleph_{\mathrm{y} 1} & \aleph_{\mathrm{y} 2} & \aleph_{\mathrm{y} 3} & \ldots \ldots . & \aleph_{\mathrm{yn}} \\
\aleph_{\mathrm{z} 1} & \aleph_{\mathrm{z} 2} & \aleph_{\mathrm{z} 3} & \ldots \ldots . & \aleph_{\mathrm{zn}}
\end{array}\right]\left[\begin{array}{c}
\mathrm{X}_{1} \\
\mathrm{X}_{2} \\
\mathrm{X}_{3} \\
\vdots \\
\mathrm{X}_{\mathrm{n}}
\end{array}\right]
$$

Equation 21 above is the general equation of the system in equilibrium applied to all the nodes of the contact flow region to formulate the global matrix equation thus;

Considering [15] shape function for a linear triangular element, we will have an element equation as shown below thus;

$$
\frac{A^{2}}{\mathrm{X}_{w} \mathrm{~L}}\left[\begin{array}{ccc}
1 & -1 & 1 \\
-1 & 1 & -1 \\
1 & -1 & 1
\end{array}\right]\left\{\begin{array}{l}
\mathrm{X}_{1} \\
\mathrm{X}_{2} \\
\mathrm{X}_{3}
\end{array}\right\}=\mathrm{f}_{(\mathrm{x})}\{\mathrm{H}\}
$$

Or;

$$
[\aleph]\left\{\mathrm{X}_{\mathrm{n}}\right\}=\{\mathrm{H}\}
$$

Equation 22 is to be applied to all the elements of the finite element mesh of Fig.4 of the contact zone, thus;

Element 1; nodes 1, 2, and 5,

$$
\frac{A^{2}}{\gamma_{w} L}\left[\begin{array}{ccc}
\aleph_{\mathrm{x} 1} & -\aleph_{\mathrm{x} 2} & \aleph_{\mathrm{x} 5} \\
-\aleph_{\mathrm{y} 1} & \aleph_{\mathrm{y} 2} & -\aleph_{\mathrm{y} 5} \\
\aleph_{\mathrm{z} 1} & -\aleph_{\mathrm{z} 2} & \aleph_{\mathrm{z} 5}
\end{array}\right]\left[\begin{array}{l}
\mathrm{X}_{1} \\
\mathrm{X}_{2} \\
\mathrm{X}_{5}
\end{array}\right]=\mathrm{f}_{(\mathrm{x})}\{\mathrm{H}\}
$$

Element 2; nodes 1, 4, and 5,

$\frac{A^{2}}{\gamma_{w} L}\left[\begin{array}{ccc}\aleph_{\mathrm{x} 1} & -\aleph_{\mathrm{x} 4} & \aleph_{\mathrm{x} 5} \\ -\aleph_{\mathrm{y} 1} & \aleph_{\mathrm{y} 4} & -\aleph_{\mathrm{y} 5} \\ \aleph_{\mathrm{z} 1} & -\aleph_{\mathrm{z} 4} & \aleph_{\mathrm{z} 5}\end{array}\right]\left[\begin{array}{l}\mathrm{X}_{1} \\ \mathrm{X}_{4} \\ \mathrm{X}_{5}\end{array}\right]=\mathrm{f}_{(\mathrm{x})}\{\mathrm{H}\}$

Element 3; nodes 4, 5, and 7,

$$
\frac{A^{2}}{\gamma_{w} L}\left[\begin{array}{ccc}
\kappa_{x 4} & -\aleph_{x 5} & \aleph_{x 7} \\
-\aleph_{y 4} & \aleph_{y 5} & -\aleph_{y 7} \\
\aleph_{z 4} & -\aleph_{z 5} & \aleph_{z 7}
\end{array}\right]\left[\begin{array}{l}
X_{4} \\
X_{5} \\
X_{7}
\end{array}\right]=f_{(x)}\{H\}
$$

Element 4; nodes 5, 7, and 8,

$$
\frac{A^{2}}{\gamma_{w} L}\left[\begin{array}{ccc}
\aleph_{x 5} & -\aleph_{x 7} & \aleph_{x 8} \\
-\aleph_{y 5} & \aleph_{y 7} & -\aleph_{y 8} \\
\aleph_{z 5} & -\aleph_{z 7} & \aleph_{z 8}
\end{array}\right]\left[\begin{array}{l}
X_{5} \\
X_{7} \\
X_{8}
\end{array}\right]=f_{(x)}\{H\}
$$

Element 5; nodes 5, 8, and 9,

$$
\frac{A^{2}}{\gamma_{w} L}\left[\begin{array}{ccc}
\aleph_{x 5} & -\aleph_{x 8} & \aleph_{x 9} \\
-\aleph_{y 5} & \aleph_{y 8} & -\aleph_{y 9} \\
\aleph_{z 5} & -\aleph_{z 8} & \aleph_{z 9}
\end{array}\right]\left[\begin{array}{l}
X_{5} \\
X_{8} \\
X_{9}
\end{array}\right]=f_{(x)}\{H\}
$$

Element 6; nodes 5, 6, and 9,

$$
\frac{A^{2}}{\gamma_{w} L}\left[\begin{array}{ccc}
\aleph_{x 5} & -\aleph_{x 6} & \aleph_{x 9} \\
-\aleph_{y 5} & \aleph_{y 6} & -\aleph_{y 9} \\
\aleph_{z 5} & -\aleph_{z 6} & \aleph_{z 9}
\end{array}\right]\left[\begin{array}{l}
X_{5} \\
X_{6} \\
X_{9}
\end{array}\right]=f_{(x)}\{H\}
$$

Element 7; nodes 3, 5, and 6,

$$
\frac{A^{2}}{\gamma_{w} L}\left[\begin{array}{ccc}
\aleph_{\mathrm{x} 3} & -\aleph_{\mathrm{x} 5} & \aleph_{\mathrm{x} 6} \\
-\aleph_{\mathrm{y} 3} & \aleph_{\mathrm{y} 5} & -\aleph_{\mathrm{y} 6} \\
\aleph_{\mathrm{z} 3} & -\aleph_{\mathrm{z} 5} & \aleph_{\mathrm{z} 6}
\end{array}\right]\left[\begin{array}{l}
\mathrm{X}_{3} \\
\mathrm{X}_{5} \\
\mathrm{X}_{6}
\end{array}\right]=\mathrm{f}_{(\mathrm{x})}\{\mathrm{H}\}
$$

Element 8; nodes 2, 3, and 5 ,

$$
\frac{A^{2}}{\gamma_{w} L}\left[\begin{array}{ccc}
\aleph_{x 2} & -\aleph_{x 3} & \aleph_{x 5} \\
-\aleph_{y 2} & \aleph_{y 3} & -\aleph_{y 5} \\
\aleph_{z 2} & -\aleph_{z 3} & \aleph_{z 5}
\end{array}\right]\left[\begin{array}{l}
X_{2} \\
X_{3} \\
X_{5}
\end{array}\right]=f_{(x)}\{H\}
$$

Finally the global matrix equation for the equilibrium condition of the studied region applying the boundary conditions as stated in Equations 32, 33, 34 and 35 is as shown in Eq.36 thus;

$$
\begin{aligned}
& { }^{5} \aleph_{\text {odd }}=0 \\
& \aleph_{5}=1 \\
& 0<\aleph_{\text {even }}<1 \\
& 0<\mathrm{X}<\mathrm{L}
\end{aligned}
$$

Assembling all the element equations applying the boundary conditions gives (56). 


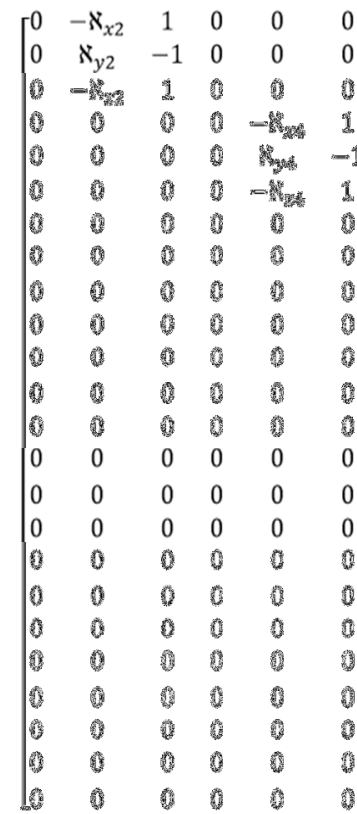

\begin{tabular}{|c|c|c|c|c|c|}
\hline 0 & 0 & 0 & 0 & 0 & 0 \\
\hline 0 & 0 & 0 & 0 & 0 & 0 \\
\hline 1) & 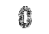 & 8 & 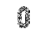 & 0 & 1 \\
\hline 知 & 6 & 0 & 5 & 1 & 篗 \\
\hline 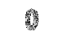 & 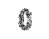 & 9 & 0 & 0 & 0 \\
\hline 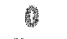 & 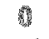 & 0 & 9 & 1 & 9 \\
\hline 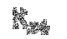 & $=x$ & (5 & 0 & 9 & 9 \\
\hline$=-\mathbb{M}_{y}$ & 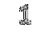 & o & 晸 & 0 & 策 \\
\hline 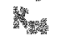 & $\sigma d$ & 1 & 6 & 4 & 4 \\
\hline 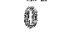 & 0 & 1 & 1 & 9 & 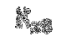 \\
\hline 䟿 & 0 & 6 & $=1$ & 0 & $=x^{\prime}$ \\
\hline 5 & 站 & 6 & 4 & 0 & 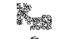 \\
\hline 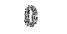 & 0 & 1 & 0 & 9 & 1 \\
\hline 0 & 0 & 0 & 0 & 0 & 0 \\
\hline 0 & 0 & 0 & 0 & 0 & 0 \\
\hline 0 & 0 & 0 & 0 & 0 & 0 \\
\hline 0 & 0 & 0 & 0 & 0 & 0 \\
\hline 0 & 0 & 0 & 0 & 0 & 0 \\
\hline 5 & 0 & 0 & 0 & 0 & 0 \\
\hline 茼 & 6 & 1) & 0 & 0 & (4) \\
\hline 4 & 0 & 0 & 6 & 商 & 籆 \\
\hline 露 & 8 & 6 & 0 & 5 & w \\
\hline a & 1 & 0 & th & 0 & 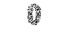 \\
\hline 喽 & 0 & 1 & 0 & 0 & 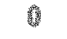 \\
\hline
\end{tabular}

\begin{tabular}{|c|c|c|c|c|c|c|c|}
\hline 0 & 0 & 0 & 0 & 0 & 0 & 0 & 0 \\
\hline 0 & 0 & 0 & 0 & 0 & 0 & 0 & 0 \\
\hline H & 留 & 1 & 1 & 0 & 0 & 0 & 0 \\
\hline 6 & 5 & 新 & 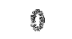 & 0 & 1 & 6 & 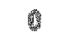 \\
\hline 1 & $\sqrt{1}$ & 籍 & 0 & 9 & 0 & 0 & tit \\
\hline 0 & 9 & $\$$ & 0 & a & 0 & 0 & 0 \\
\hline 0 & 19 & 6 & (⿻) & 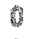 & 6 & 0 & 9 \\
\hline V & 0 & 0 & 0 & 0 & 0 & 6 & 6 \\
\hline 9 & (1) & 1 & 0 & 9 & 9 & 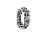 & 9 \\
\hline 8 & 6 & I) & 0 & 0 & (1) & 20 & 0 \\
\hline (3) & 0 & 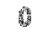 & 0 & 0 & 0 & 9 & 6 \\
\hline 0 & 0 & 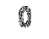 & (a) & 1 & 0 & W & 0 \\
\hline$-x^{3}$ & 0 & 0 & 0 & 1 & (1) & 1 & 1 \\
\hline$\aleph_{y 8}$ & 0 & 0 & 0 & 0 & 0 & 0 & 0 \\
\hline$-\kappa_{z 8}$ & 0 & 0 & 0 & 0 & 0 & 0 & 0 \\
\hline 0 & 0 & 1 & $-\aleph_{x 6}$ & 0 & 0 & 0 & 0 \\
\hline 9 & 0 & -1 & 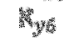 & 0 & 0 & 0 & 0 \\
\hline 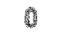 & 0 & 1 & $=\mathrm{x}_{\mathrm{K}}$ & 0 & 0 & 0 & 0 \\
\hline 0 & 9 & 0 & 0 & 0 & 0 & -1 & 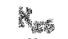 \\
\hline 6 & 0 & 1 & 6 & 0 & 5 & $\mathbb{1}$ & $=$ and \\
\hline 0 & 部 & 1 & 1 & 0 & 0 & -1 & 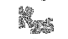 \\
\hline th & 4 & 3 & 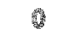 & 0 & $\sqrt{4}$ & 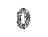 & (6) \\
\hline 0 & 0 & 0 & 0 & 0 & 1 & $\theta$ & 0 \\
\hline G & 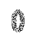 & 9 & 0 & 0 & 6 & 0 & 1 \\
\hline
\end{tabular}

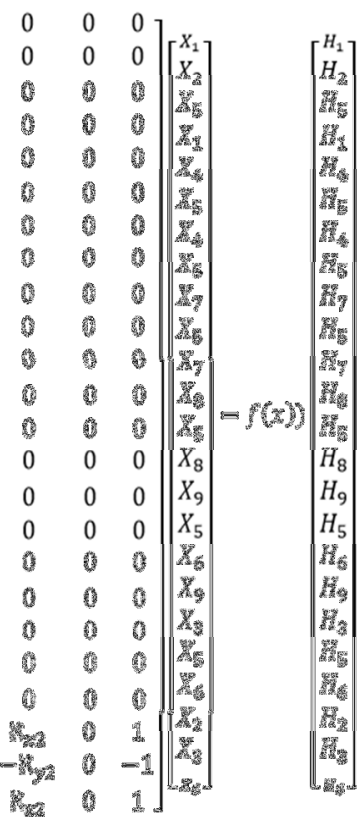

Solving Equation 36 gives equation (57):

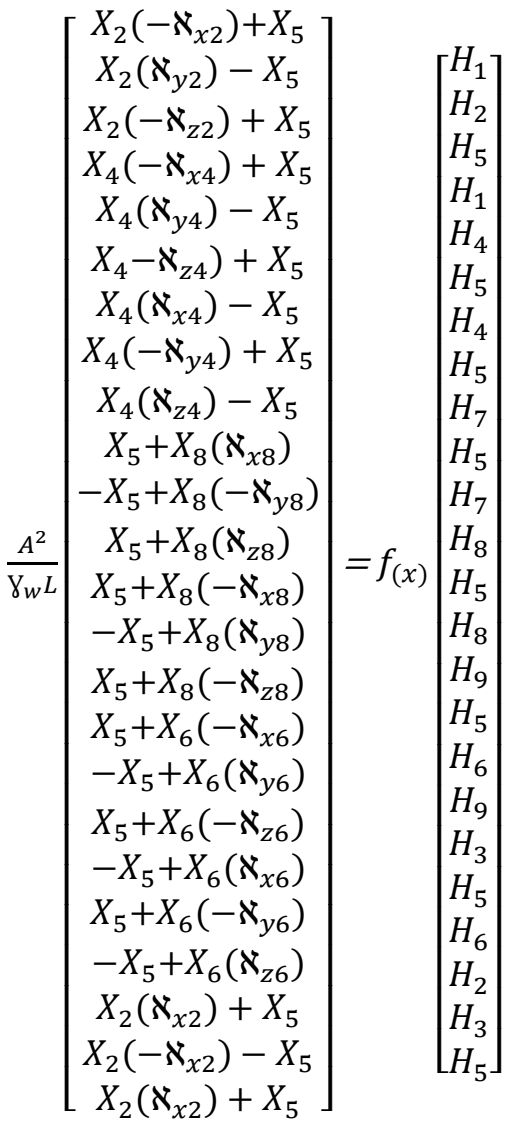

Furthermore,

$$
\begin{aligned}
H_{(x)}=\frac{A^{2}}{\gamma_{w} L}\{[ & \left.X_{2}\left(-\aleph_{x 2}\right)+X_{5}\right]+\left[X_{2}\left(\aleph_{y 2}\right)-X_{5}\right] \\
& +\left[X_{2}\left(-\aleph_{z 2}\right)+X_{5}\right]+\left[X_{4}\left(-\aleph_{x 4}\right)+X_{5}\right] \\
& \left.+\left[X_{4}\left(\aleph_{y 4}\right)-X_{5}\right]+\left[X_{4}-\aleph_{z 4}\right)+X_{5}\right] \\
& +\left[X_{4}\left(\aleph_{x 4}\right)-X_{5}\right]+\left[X_{4}\left(-\aleph_{y 4}\right)+X_{5}\right] \\
& +\left[X_{4}\left(\aleph_{z 4}\right)-X_{5}\right]+\left[X_{5}+X_{8}\left(\aleph_{x 8}\right)\right] \\
& +\left[-X_{5}+X_{8}\left(-\aleph_{y 8}\right)\right]+\left[X_{5}+X_{8}\left(\aleph_{z 8}\right)\right] \\
& +\left[X_{5}+X_{8}\left(-\aleph_{x 8}\right)\right]+\left[-X_{5}+X_{8}\left(\aleph_{y 8}\right)\right] \\
& +\left[X_{5}+X_{8}\left(-\aleph_{z 8}\right)\right]+\left[X_{5}+X_{6}\left(-\aleph_{x 6}\right)\right] \\
& +\left[-X_{5}+X_{6}\left(\aleph_{y 6}\right)\right]+\left[X_{5}+X_{6}\left(-\aleph_{z 6}\right)\right] \\
& +\left[-X_{5}+X_{6}\left(\aleph_{x 6}\right)\right]+\left[X_{5}+X_{6}\left(-\aleph_{y 6}\right)\right] \\
& +\left[-X_{5}+X_{6}\left(\aleph_{z 6}\right)\right]+\left[X_{2}\left(\aleph_{x 2}\right)+X_{5}\right] \\
& +\left[X_{2}\left(-\aleph_{x 2}\right)-X_{5}\right] \\
& \left.+\left[X_{2}\left(\aleph_{x 2}\right)+X_{5}\right]\right\}
\end{aligned}
$$

Collecting like terms and solving same would give;

$$
H_{(x)}=\frac{4 X_{5} \aleph_{x 2} A^{2}}{\gamma_{w} L}=\frac{4 \pi^{2} r^{4} X_{5} \aleph_{x 2}}{\gamma_{w} L}
$$

$\mathrm{r}$ is the average radius of the discrete soil particles $=0.002857 \mathrm{~m}$

$\mathrm{X}_{5}$ is the flow distance and this varies between 0.6, $1.2,1.8 \ldots . . .$. , and 6.0

$\aleph_{x_{2}}$ is the equilibrium stress of the system which factors vary between $0.1,0.2,0.3 \ldots, 1.0$ [2]

$\gamma_{w}$ is the unit weight of water $=1000 \mathrm{~kg} / \mathrm{m}^{3}$

$\mathrm{L}$ is the cross sectional length of the flow medium $=$ $6 \mathrm{~m}$.

Substituting for values in Equation 39, we would have the model equation for the head restoring equilibrium at well pumping thus;

$$
H_{(x)}=0.0065814 . r^{4} X_{5} \aleph_{x_{2}}
$$




\subsection{Laboratory Investigation Model}

The geophysical laboratory investigation was carried out on the sample collected from borehole sites located within Umuahia [16] where there have been records of failed boreholes at the aquifer depth of 50 to 68 meters located on latitude North $5^{\circ} 3^{I} 32.80^{I I}$ and longitude East $7^{\circ} 29^{I} 46^{I I}$ with average rainfall of between $2000 \mathrm{~mm}$ to $2500 \mathrm{~mm}$ [17].(a) the casagrande apparatus was used to carry out the Atterberg limit test with free falling cup from a height equal to $10 \pm 0.2 \mathrm{~mm}$ above the base [18], (b) the standard proctor compaction was also used to determine the optimum moisture content, maximum dry density, and specific gravity of the sample [18; 19], (c)The constant head permeameter was used to determine the permeability coefficient (hydraulic Conductivity), K of the sample $[18 ; 20]$ and (d) a direct shear box was used to establish the normal stress at which the sample was sheared [18].And finally, prototype well failure test was conducted as shown in Figure 6. At the same time a power regulator of 10 voltage speeds was fabricated to power the submersible pump at 10 different voltages supplied between 150 volts and 240 volts. This well (Fig.6) was filled with the granular soil sample from already drilled boreholes and allowed to compact naturally for a period of 8 months from April to December, 2014. After this sufficient time has been allowed for the setup to achieve compaction, a well was bored through the sample to a depth of 6 meters. Furthermore, a local 10 lever voltage control was fabricated with potential differences of $150,160,170$, $180,190,200,210,220,230$ and 240 volts to supply pumping power to the submersible pump to yield at 10 distinct discharge rates, q. However a one horse power submersible pump was hired for this experimental operation and the attendant PVC perforated pipes and riser pipes.

The mathematical expression of the foregoing is as follows;

$$
\text { Power, } \mathrm{P}_{\mathrm{o}}=\text { pressure, } \rho \mathrm{x} \text { flow rate, } \mathrm{q}=\rho \mathrm{q}
$$
Also,

$$
\mathrm{P}_{\mathrm{o}}=\text { Current, } \mathrm{I} \mathrm{x} \text { potential difference, } \mathrm{V}
$$

This implies that,

$$
\begin{aligned}
& \rho q=I V \\
& q=\frac{I V}{\rho}
\end{aligned}
$$

But the pressure of the set up,

$$
\rho=\mathrm{mgh}
$$

$=58860 \mathrm{~N} / \mathrm{m}^{2}$

Therefore,

$$
q=\frac{13 \mathrm{~V}}{58860}=0.000221 \mathrm{~V}
$$

Furthermore, the power generated by the supply system is;

$$
P_{o}=58.860 q
$$

However, the results of the laboratory examination from the well failure test are as tabulated below in Table 2;

Finally, the governing equation relevant to the laboratory implementation of the present research work which also evolved from fundamental equations is such that the head at which equilibrium is restored $\mathrm{h}_{\mathrm{R}}$ as stated below thus,

$$
h_{R}=K \frac{\sigma A L}{q \cdot \gamma_{w}}
$$

\section{RESULTS AND DISCUSSION}

The result of the geophysical examination carried out on the sample under study is as tabulated in Table 1. Table 2 shows the results of the borehole prototype well failure test.

Equation 30 was solved with the relevant boundary conditions to generate the results as shown in Table 3 and Figure 7 below thus;

Recall that the restoring hydraulic head of the system was deduced from the mathematical model as;

$H_{(x)}=0.0065814 . r^{4} X_{5} \kappa_{x_{2}}$, where;

$r$ is the average radius of the discrete soil particles $=0.002857 \mathrm{~m}$

$\mathrm{X}_{5}$ is the flow distance and this varies between $0.6,1.2$, 1.8..., and 6.0. and $\aleph_{x_{2}}$ is the equilibrium stress of the system which factors vary between $0.1,0.2,0.3 \ldots ., 1.0$ and the matlab solution of the above equation is as shown below in Table 4 and Fig. 8.

From the foregoing, it could be deduced and established that the head causing critical seepage which consequently causes dislodgement of particles is expressed as $h_{(x)}=0.000524 r^{2}\left[X_{5}\left(5-2 S F_{x_{2}}\right)\right]$ as shown in Eq.20 generated from the element model. Tables 3 and 4 and Figures 7 and 8 have shown that there is strong agreement between the mathematical model and the laboratory study with closest agreement at the flow distance of $1.8 \mathrm{~m}$ compared to the lab investigation. 


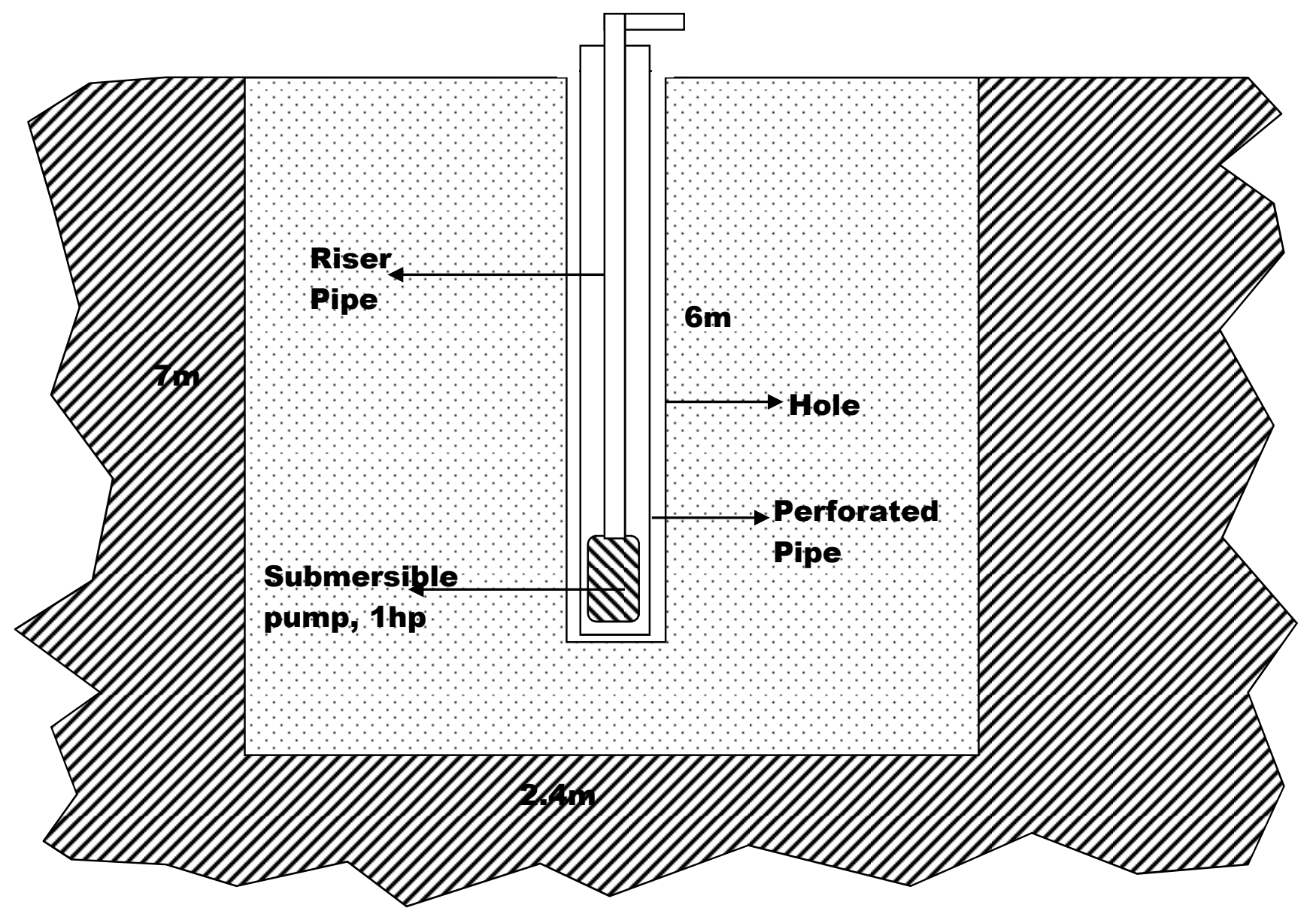

Figure 6: Prototype well failure test setup

Table1: Geophysical properties of soil sample under study [20; 21]

\begin{tabular}{|c|c|c|c|}
\hline Parameter & Result & Parameter & Result \\
\hline Liquid Limit & 14.00 & OMC & $7.075 \%$ \\
\hline Plastic Limit & 6.67 & Specific Gravity G & 2.857 \\
\hline Plasticity Index & 7.33 & Proven Ring Factor k & $0.004105 \mathrm{KN} / \mathrm{div}$ \\
\hline $\mathrm{Cu}$ & 6.79 & Area of Shear Box & $0.01 \mathrm{~m}^{2}$ \\
\hline $\mathrm{Cc}$ & 1.52 & Normal Stress $\sigma$ & $10.275 \mathrm{KN} / \mathrm{m}^{2}$ \\
\hline Classification(AASHTO) & $A-2-4$ & Frictional angle & $48^{\circ}$ \\
\hline Grading & Well graded & Cohesion & $40 \mathrm{KN}$ \\
\hline MDD & $1.84 \mathrm{mg} / \mathrm{m}^{3}$ & Soil Type & Gravel and sand \\
\hline $\mathrm{X}_{\text {sat }}$ & $19.26 \mathrm{KN} / \mathrm{m}^{3}$ & $\mathrm{x}_{\mathrm{w}}$ & $9.8 \mathrm{KN} / \mathrm{m}^{3}$ \\
\hline $\mathrm{x}_{\mathrm{b}}$ & $9.46 \mathrm{KN} / \mathrm{m}^{3}$ & $\mathrm{i}_{\mathrm{c}}$ & 0.9653 \\
\hline K & $3.0806 \mathrm{E}-11 \mathrm{cl}$ & & \\
\hline
\end{tabular}

Table 2: Prototype well failure test result and critical hydraulic head

\begin{tabular}{lll}
\hline Voltage & Pump discharge, $\mathrm{q}\left(\mathrm{m}^{3} / \mathrm{s}\right)$ & Generated pump power, $\mathrm{P}_{\mathrm{o}}(\mathrm{hp})$ \\
\hline 150 & 0.03313 & 1.95 \\
160 & 0.03536 & 2.08 \\
170 & 0.03757 & 2.21 \\
180 & 0.03978 & 2.34 \\
190 & 0.04199 & 2.47 \\
200 & 0.04420 & 2.60 \\
210 & 0.04641 & 2.73 \\
220 & 0.04862 & 2.86 \\
230 & 0.05083 & 2.99 \\
240 & 0.05304 & 3.12 \\
\hline
\end{tabular}


Table 3: Critical hydraulic head and seepage force model

\begin{tabular}{|l|l|l|l|l|l|l|l|l|l|l|l|}
\hline \multirow{3}{*}{$S_{x_{x_{2}}}$} & \multicolumn{8}{|c|}{ Critical hydraulic head $h_{(x)}=0.000524 r^{2}\left[X_{5}\left(5-2 S F_{x_{2}}\right)\right] @$} & $\begin{array}{l}\text { Lab. } \\
\text { Critical } \\
\text { head, } \\
h_{c}\end{array}$ \\
\cline { 2 - 15 } & 0.6 & 1.2 & 1.8 & 2.4 & 3.0 & 3.6 & 4.2 & 4.8 & 5.4 & 6.0 \\
\hline 0.1 & $.123 \mathrm{E}-7$ & $.247 \mathrm{E}-7$ & $.370 \mathrm{E}-7$ & $.493 \mathrm{E}-7$ & $.616 \mathrm{E}-7$ & $.739 \mathrm{E}-7$ & $.862 \mathrm{E}-7$ & $.985 \mathrm{E}-7$ & $1.11 \mathrm{E}-7$ & $1.23 \mathrm{E}-7$ & $.327 \mathrm{E}-7$ \\
\hline 0.2 & $.118 \mathrm{E}-7$ & $.236 \mathrm{E}-7$ & $.354 \mathrm{E}-7$ & $.472 \mathrm{E}-7$ & $.590 \mathrm{E}-7$ & $.709 \mathrm{E}-7$ & $.826 \mathrm{E}-7$ & $.944 \mathrm{E}-7$ & $1.06 \mathrm{E}-7$ & $1.18 \mathrm{E}-7$ & $.313 \mathrm{E}-7$ \\
\hline 0.3 & $.113 \mathrm{E}-7$ & $.226 \mathrm{E}-7$ & $.339 \mathrm{E}-7$ & $.451 \mathrm{E}-7$ & $.565 \mathrm{E}-7$ & $.677 \mathrm{E}-7$ & $.790 \mathrm{E}-7$ & $.904 \mathrm{E}-7$ & $1.02 \mathrm{E}-7$ & $1.13 \mathrm{E}-7$ & $.299 \mathrm{E}-7$ \\
\hline 0.4 & $.108 \mathrm{E}-7$ & $.215 \mathrm{E}-7$ & $.323 \mathrm{E}-7$ & $.431 \mathrm{E}-7$ & $.539 \mathrm{E}-7$ & $.646 \mathrm{E}-7$ & $.754 \mathrm{E}-7$ & $.862 \mathrm{E}-7$ & $.970 \mathrm{E}-7$ & $1.08 \mathrm{E}-7$ & $.286 \mathrm{E}-7$ \\
\hline 0.5 & $.103 \mathrm{E}-7$ & $.206 \mathrm{E}-7$ & $.308 \mathrm{E}-7$ & $.411 \mathrm{E}-7$ & $.513 \mathrm{E}-7$ & $.616 \mathrm{E}-7$ & $.718 \mathrm{E}-7$ & $.821 \mathrm{E}-7$ & $.924 \mathrm{E}-7$ & $1.03 \mathrm{E}-7$ & $.272 \mathrm{E}-7$ \\
\hline 0.6 & $.097 \mathrm{E}-7$ & $.195 \mathrm{E}-7$ & $.292 \mathrm{E}-7$ & $.390 \mathrm{E}-7$ & $.487 \mathrm{E}-7$ & $.585 \mathrm{E}-7$ & $.682 \mathrm{E}-7$ & $.780 \mathrm{E}-7$ & $.877 \mathrm{E}-7$ & $.975 \mathrm{E}-7$ & $.259 \mathrm{E}-7$ \\
\hline 0.7 & $.092 \mathrm{E}-7$ & $.184 \mathrm{E}-7$ & $.277 \mathrm{E}-7$ & $.370 \mathrm{E}-7$ & $.462 \mathrm{E}-7$ & $.554 \mathrm{E}-7$ & $.646 \mathrm{E}-7$ & $.739 \mathrm{E}-7$ & $.832 \mathrm{E}-7$ & $.924 \mathrm{E}-7$ & $.245 \mathrm{E}-7$ \\
\hline 0.8 & $.087 \mathrm{E}-7$ & $.175 \mathrm{E}-7$ & $.262 \mathrm{E}-7$ & $.349 \mathrm{E}-7$ & $.436 \mathrm{E}-7$ & $.523 \mathrm{E}-7$ & $.611 \mathrm{E}-7$ & $.698 \mathrm{E}-7$ & $.785 \mathrm{E}-7$ & $.873 \mathrm{E}-7$ & $.232 \mathrm{E}-7$ \\
\hline 0.9 & $.082 \mathrm{E}-7$ & $.164 \mathrm{E}-7$ & $.247 \mathrm{E}-7$ & $.328 \mathrm{E}-7$ & $.411 \mathrm{E}-7$ & $.493 \mathrm{E}-7$ & $.575 \mathrm{E}-7$ & $.657 \mathrm{E}-7$ & $.739 \mathrm{E}-7$ & $.821 \mathrm{E}-7$ & $.218 \mathrm{E}-7$ \\
\hline 1.0 & $.077 \mathrm{E}-7$ & $.154 \mathrm{E}-7$ & $.231 \mathrm{E}-7$ & $.308 \mathrm{E}-7$ & $.385 \mathrm{E}-7$ & $.462 \mathrm{E}-7$ & $.539 \mathrm{E}-7$ & $.616 \mathrm{E}-7$ & $.693 \mathrm{E}-7$ & $.770 \mathrm{E}-7$ & $.204 \mathrm{E}-7$ \\
\hline
\end{tabular}

Table 4: Restoring hydraulic head and equilibrium stress model

\begin{tabular}{|c|c|c|c|c|c|c|c|c|c|c|c|}
\hline \multirow[t]{2}{*}{$\aleph_{x_{2}}$} & \multicolumn{10}{|c|}{$\begin{array}{l}\text { Restoring hydraulic head } H_{(x)}=0.0065814 . r^{4} X_{5} \aleph_{x_{2}} @ \\
X_{5} \text { equals }\end{array}$} & \multirow{2}{*}{$\begin{array}{l}\text { Lab. } \\
\text { Restoring } \\
\text { head, } h_{R}\end{array}$} \\
\hline & 0.6 & 1.2 & 1.8 & 2.4 & 3.0 & 3.6 & 4.2 & 4.8 & 5.4 & 6.0 & \\
\hline 0.1 & $.27 \mathrm{E}-13$ & $.53 \mathrm{E}-13$ & $.79 \mathrm{E}-13$ & $1.1 \mathrm{E}-13$ & $1.3 \mathrm{E}-13$ & $1.6 \mathrm{E}-13$ & $1.8 \mathrm{E}-13$ & $2.1 \mathrm{E}-13$ & $2.4 \mathrm{E}-13$ & $2.6 \mathrm{E}-13$ & $1.5 \mathrm{E}-13$ \\
\hline 0.2 & $.53 \mathrm{E}-13$ & $1.1 \mathrm{E}-13$ & $1.6 \mathrm{E}-13$ & $2.1 \mathrm{E}-13$ & $2.6 \mathrm{E}-13$ & $3.2 \mathrm{E}-13$ & $3.7 \mathrm{E}-13$ & $4.2 \mathrm{E}-13$ & $4.8 \mathrm{E}-13$ & $5.3 \mathrm{E}-13$ & $1.9 \mathrm{E}-13$ \\
\hline 0.3 & $.79 \mathrm{E}-13$ & $1.6 \mathrm{E}-13$ & $2.4 \mathrm{E}-13$ & $3.2 \mathrm{E}-13$ & $4.0 \mathrm{E}-13$ & $4.8 \mathrm{E}-13$ & $5.6 \mathrm{E}-13$ & $6.4 \mathrm{E}-13$ & $7.1 \mathrm{E}-13$ & $7.9 \mathrm{E}-13$ & $2.4 \mathrm{E}-13$ \\
\hline 0.4 & $1.1 \mathrm{E}-13$ & $2.1 \mathrm{E}-13$ & $3.2 \mathrm{E}-13$ & $3.6 \mathrm{E}-13$ & $5.3 \mathrm{E}-13$ & $6.4 \mathrm{E}-13$ & 7.4E-13 & $8.5 \mathrm{E}-13$ & $9.5 \mathrm{E}-13$ & $10.6 \mathrm{E}-13$ & $3.1 \mathrm{E}-13$ \\
\hline 0.5 & $1.3 \mathrm{E}-13$ & $2.6 \mathrm{E}-13$ & $4.0 \mathrm{E}-13$ & $5.3 \mathrm{E}-13$ & $6.6 \mathrm{E}-13$ & $7.9 \mathrm{E}-13$ & $9.3 \mathrm{E}-13$ & $10.6 \mathrm{E}-13$ & 11.9E-13 & $13.2 \mathrm{E}-13$ & $3.9 \mathrm{E}-13$ \\
\hline 0.6 & $1.6 \mathrm{E}-13$ & $3.2 \mathrm{E}-13$ & $4.8 \mathrm{E}-13$ & $6.4 \mathrm{E}-13$ & $7.9 \mathrm{E}-13$ & $9.5 \mathrm{E}-13$ & $11.1 \mathrm{E}-13$ & $12.7 \mathrm{E}-13$ & 14.3E-13 & $15.9 \mathrm{E}-13$ & $4.6 \mathrm{E}-13$ \\
\hline 0.7 & $1.8 \mathrm{E}-13$ & $3.7 \mathrm{E}-13$ & $5.6 \mathrm{E}-13$ & $7.4 \mathrm{E}-13$ & $9.3 \mathrm{E}-13$ & $11.1 \mathrm{E}-13$ & $13.0 \mathrm{E}-13$ & $14.8 \mathrm{E}-13$ & $16.7 \mathrm{E}-13$ & $18.5 \mathrm{E}-13$ & $4.8 \mathrm{E}-13$ \\
\hline 0.8 & $2.1 \mathrm{E}-13$ & $4.2 \mathrm{E}-13$ & $6.4 \mathrm{E}-13$ & $8.5 \mathrm{E}-13$ & $10.6 \mathrm{E}-13$ & $12.7 \mathrm{E}-13$ & $14.8 \mathrm{E}-13$ & $16.9 \mathrm{E}-13$ & $19.0 \mathrm{E}-13$ & $21.2 \mathrm{E}-13$ & $5.5 \mathrm{E}-13$ \\
\hline 0.9 & $2.4 \mathrm{E}-13$ & $4.8 \mathrm{E}-13$ & $7.1 \mathrm{E}-13$ & $9.5 \mathrm{E}-13$ & $11.9 \mathrm{E}-13$ & $14.3 \mathrm{E}-13$ & $16.7 \mathrm{E}-13$ & $19.0 \mathrm{E}-13$ & $21.4 \mathrm{E}-13$ & $23.8 \mathrm{E}-13$ & $6.4 \mathrm{E}-13$ \\
\hline 1.0 & $2.7 \mathrm{E}-13$ & $5.3 \mathrm{E}-13$ & $7.9 \mathrm{E}-13$ & $10.6 \mathrm{E}-13$ & $13.2 \mathrm{E}-13$ & 15.9E-13 & $18.5 \mathrm{E}-13$ & $21.2 \mathrm{E}-13$ & $23.8 \mathrm{E}-13$ & $26.5 \mathrm{E}-13$ & $7.2 \mathrm{E}-13$ \\
\hline
\end{tabular}

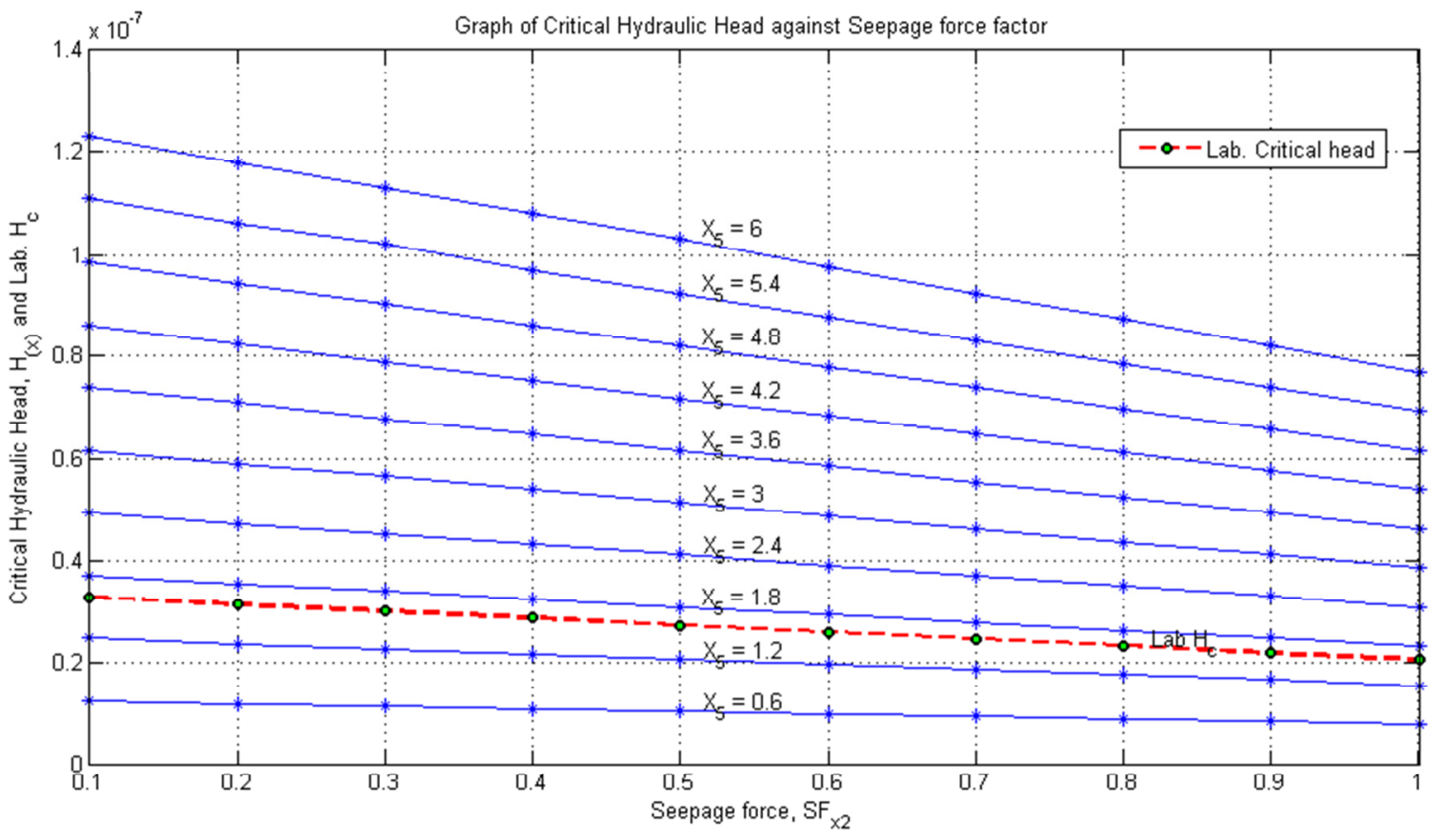

Figure 7: Numerical and laboratory critical hydraulic head of system 
\%Table 5: Restoring hydraulic head and equilibrium stress model

$\% \quad$ N_x2 Restoring hydraulic head $\mathrm{h}_{-}(\mathrm{x}) @$ Lab. Restoring head

$\% \quad$ X_5 equals (column 2 to 11 ) H_R (last column)

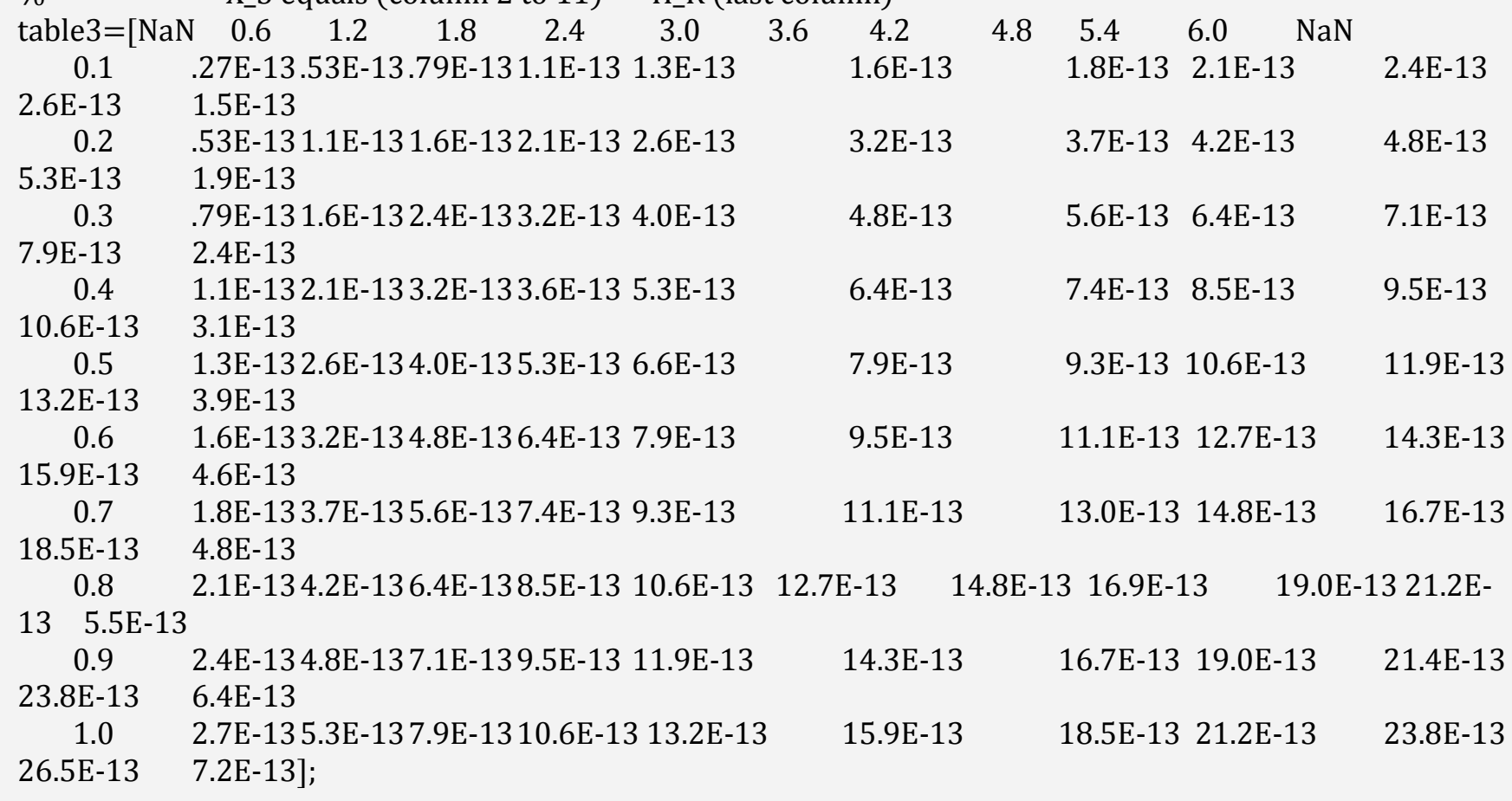

\%2. Plot heads ( $\mathrm{H}(\mathrm{x})$ and $\mathrm{hR}$ ) versus stress factors (N_(x_2 )) from Table 3

$\mathrm{Nx}$ = table3(2:end,1);

figure(2);

for $\mathrm{n}=1: 11$

Head $=$ table3 $(2:$ end, $n+1)$;

if $\mathrm{n}<=10$

plot(Nx2,Head,'*-');

else gtext(['X_5 = ' num2str(table2(1,n+1))]);

h2=plot(Nx2,Head,'--ro','LineWidth',2,...

'MarkerEdgeColor','k',...

'MarkerFaceColor','g',...

'MarkerSize',5);

end gtext('Lab H_R');

hold on;

end

legend(h2,'Lab. Restoring head','Location','Best')

grid on; xlabel('Stress Factor, $\backslash$ aleph_ $\{\mathrm{x} 2\}$ (in metres)');

ylabel('Restoring Hydraulic Head, $H_{-}\{(x)\}$ and Lab. H_R');

title('Graph of Restoring Hydraulic Head against Stress Factor');

hold off

From Fig.7, it can be deduced that a decrease in the critical hydraulic head causing critical seepage is accompanied with an increase in seepage force which is evidence that dislodgement of particles increases the geometry of the channel of flow thereby increasing seepage to a critical point with its attendant quicksand effect $[2 ; 3]$.
Table 4 also show that increase in flow distance increases the hydraulic head considerably and Table 2 has also shown that increase in voltage increases critical hydraulic head which in turn causes quicksand effect.

The hydraulic head restoring equilibrium between contact force (inter-granular force) and seepage force is deduced as per Equation 40 as 
$H_{(x)}=0.0065814 . r^{4} X_{5} \aleph_{x_{2}}$ from the mathematical model and values are seen to be less than the heads causing boiling, (Tables 3 and 4), which proves the positive effect of inter-granular force in the system.

There is strong agreement between the mathematical model hydraulic heads and the laboratory model hydraulic head in that they both increased progressively and relatively with equilibrium stress of the system as shown in Table 4 and Figure 7 with the closest agreement at the flow distance of $1.8 \mathrm{~m}$.

It can also be deduced that hydraulic head increased with increase in flow distance for all the stress factors. The model chats as shown in Fig. 7 and 8 "numerical and laboratory critical hydraulic head of the system" and "numerical and laboratory restoring hydraulic heads of the system" can be used by engineers to design and monitor the performance of wells taking into account all the conditions that gave rise to the results. However, it can be deduced also that the behaviour of boreholes during its critical condition and equilibrium condition with respect to hydraulic heads, seepage force, equilibrium stress factor etc at different flow distance points within the flow region under study and at the same time considering the contact between particles.

The model plot as shown in Fig.7 shows the critical heads below which the system is safe. The region of safety has been achieved by the counter effect of the contact force on the seepage force which gave rise to the model plots as shown in Fig.7. All the head points of the model as shown in Fig.8 are safe hydraulic heads beyond which the system shifts to the critical state region which is only possible when seepage force becomes more than the contact force between particles. For purposes of future calculations and referring to the degree of agreement earlier stated between the mathematical model and laboratory investigation for the critical state, the standard critical hydraulic head of the system from Table 3 and Fig.7 is 2.92E-8 which has the strongest agreement with 2.59E-8 of the laboratory study with a deviation of 3.3E-9. Also for the equilibrium state condition, the restoring hydraulic head of the system based on the closest agreement between the mathematical and laboratory models is $4 \mathrm{E}-13$ compared to $3.9 \mathrm{E}-13$ of the laboratory result with a deviation of $1 \mathrm{E}-14$. Figure 8 has also shown that the stress factor had increased with restoring hydraulic head which shows that the stress existing or generated by particles at the interface of contact contributes to the counter effect on seepage force forestalling its critical state which is disadvantageous to the operation and performance of boreholes.

The degree of agreement between the mathematical and laboratory model is verified using the correlation analysis thus; [22]

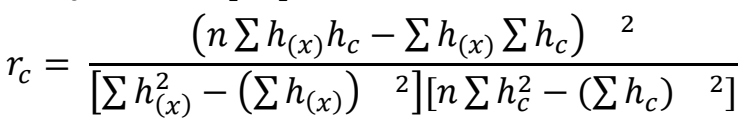

Where $r_{c}$ is the Correlation coefficient for critical state model, $h_{c}$ is the laboratory critical hydraulic head and $h_{(x)}$ is the model critical hydraulic head

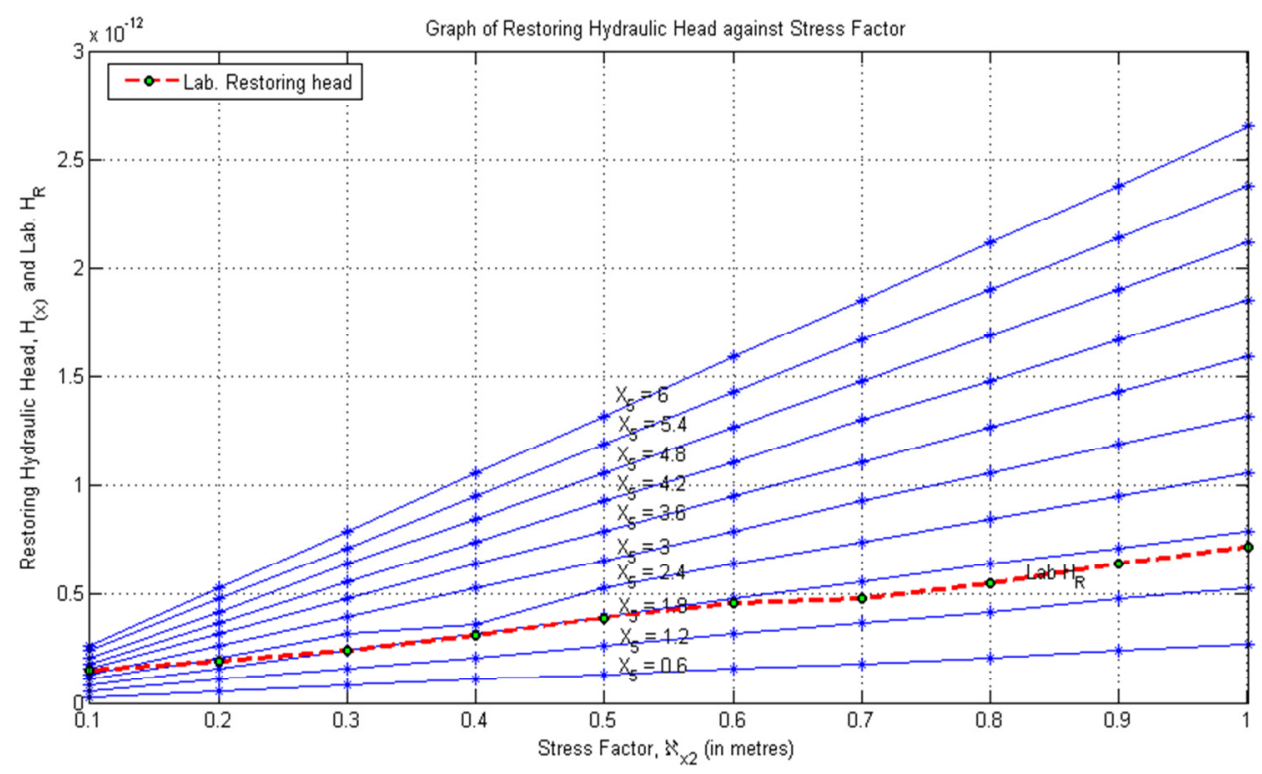

Figure 8: Equilibrium condition of the restoring hydraulic head 
Table 5: Critical State Model correlation analysis

\begin{tabular}{|c|c|c|c|c|}
\hline$h_{(x)}$ & $h_{c}$ & $h_{(x)}^{2}$ & $h_{c}^{2}$ & $h_{(x)} h_{c}$ \\
\hline $0.370 \mathrm{E}-7$ & $0.327 \mathrm{E}-7$ & $0.1369 \mathrm{E}-14$ & $0.1069 \mathrm{E}-14$ & $0.1210 \mathrm{E}-14$ \\
\hline $0.354 \mathrm{E}-7$ & $0.313 \mathrm{E}-7$ & $0.1253 \mathrm{E}-14$ & $0.0980 \mathrm{E}-14$ & $0.1108 \mathrm{E}-14$ \\
\hline $0.339 \mathrm{E}-7$ & $0.299 \mathrm{E}-7$ & $0.1149 \mathrm{E}-14$ & $0.0894 \mathrm{E}-14$ & $0.1014 \mathrm{E}-14$ \\
\hline $0.323 \mathrm{E}-7$ & $0.286 \mathrm{E}-7$ & $0.1043 \mathrm{E}-14$ & $0.0818 \mathrm{E}-14$ & $0.0924 \mathrm{E}-14$ \\
\hline $0.308 \mathrm{E}-7$ & $0.272 \mathrm{E}-7$ & $0.0949 \mathrm{E}-14$ & $0.0740 \mathrm{E}-14$ & $0.0838 \mathrm{E}-14$ \\
\hline $0.292 \mathrm{E}-7$ & $0.259 \mathrm{E}-7$ & $0.0853 \mathrm{E}-14$ & $0.0671 \mathrm{E}-14$ & $0.0756 \mathrm{E}-14$ \\
\hline $0.277 \mathrm{E}-7$ & $0.245 \mathrm{E}-7$ & $0.0767 \mathrm{E}-14$ & $0.0600 \mathrm{E}-14$ & $0.0679 \mathrm{E}-14$ \\
\hline $0.262 \mathrm{E}-7$ & $0.232 \mathrm{E}-7$ & $0.0686 \mathrm{E}-14$ & $0.0538 \mathrm{E}-14$ & $0.0608 \mathrm{E}-14$ \\
\hline $0.247 \mathrm{E}-7$ & $0.218 \mathrm{E}-7$ & $0.0610 \mathrm{E}-14$ & $0.0475 \mathrm{E}-14$ & $0.0538 \mathrm{E}-14$ \\
\hline $0.231 \mathrm{E}-7$ & $0.204 \mathrm{E}-7$ & $0.0534 \mathrm{E}-14$ & $0.0416 \mathrm{E}-14$ & $0.0471 \mathrm{E}-14$ \\
\hline
\end{tabular}

$$
\begin{gathered}
\sum h_{(x)}=3.003 \mathrm{E}-7 \\
\sum h_{c}=2.655 \mathrm{E}-7 \\
\left(\sum h_{c}\right)^{2}=0.7201 \mathrm{E}-14 \\
\left(\sum h_{(x)}\right)^{2}=0.9213 \mathrm{E}-14 \\
\sum h_{(x)} h_{c}=0.8146 \mathrm{E}-14
\end{gathered}
$$

Substituting the values of the unknowns in Equation 69 above, the correlation coefficient for the critical state model is;

$$
r_{c}=1.00975
$$

Similarly,

$$
r_{R}=\frac{\left(n \sum H_{(x)} h_{R}-\sum H_{(x)} \sum h_{R}\right)^{2}}{\left[\sum H_{(x)}^{2}-\left(\sum H_{(x)}\right)^{2}\right]\left[n \sum h_{R}^{2}-\left(\sum h_{R}\right)^{2}\right]}
$$

Where;

$r_{R}$ is the Correlation coefficient for restoring or equilibrium state model, $h_{R}$ is the laboratory restoring hydraulic head and $H_{(x)}$ is the model restoring hydraulic head

\section{Table 6: Equilibrium State Model correlation analysis}

\begin{tabular}{ccccc}
\hline$H_{(x)}$ & $h_{R}$ & $H_{(x)}^{2}$ & $h_{R}^{2}$ & $H_{(x)} h_{R}$ \\
\hline $.79 \mathrm{E}-13$ & $1.5 \mathrm{E}-13$ & $0.6241 \mathrm{E}-26$ & $2.2500 \mathrm{E}-26$ & $1.185 \mathrm{E}-26$ \\
$1.6 \mathrm{E}-13$ & $1.9 \mathrm{E}-13$ & $2.5600 \mathrm{E}-26$ & $3.6100 \mathrm{E}-26$ & $3.040 \mathrm{E}-26$ \\
$2.4 \mathrm{E}-13$ & $2.4 \mathrm{E}-13$ & $5.7600 \mathrm{E}-26$ & $5.7600 \mathrm{E}-26$ & $5.760 \mathrm{E}-26$ \\
$3.2 \mathrm{E}-13$ & $3.1 \mathrm{E}-13$ & $10.240 \mathrm{E}-26$ & $9.6100 \mathrm{E}-26$ & $9.920 \mathrm{E}-26$ \\
$4.0 \mathrm{E}-13$ & $3.9 \mathrm{E}-13$ & $16.000 \mathrm{E}-26$ & $15.210 \mathrm{E}-26$ & $15.60 \mathrm{E}-26$ \\
$4.8 \mathrm{E}-13$ & $4.6 \mathrm{E}-13$ & $23.040 \mathrm{E}-26$ & $21.160 \mathrm{E}-26$ & $22.08 \mathrm{E}-26$ \\
$5.6 \mathrm{E}-13$ & $4.8 \mathrm{E}-13$ & $31.360 \mathrm{E}-26$ & $23.040 \mathrm{E}-26$ & $26.88 \mathrm{E}-26$ \\
$6.4 \mathrm{E}-13$ & $5.5 \mathrm{E}-13$ & $40.960 \mathrm{E}-26$ & $30.250 \mathrm{E}-26$ & $35.20 \mathrm{E}-26$ \\
$7.1 \mathrm{E}-13$ & $6.4 \mathrm{E}-13$ & $50.410 \mathrm{E}-26$ & $40.960 \mathrm{E}-26$ & $45.44 \mathrm{E}-26$ \\
$7.9 \mathrm{E}-13$ & $7.2 \mathrm{E}-13$ & $62.410 \mathrm{E}-26$ & $51.840 \mathrm{E}-26$ & $56.88 \mathrm{E}-26$ \\
\hline
\end{tabular}

$$
\begin{gathered}
\sum H_{(x)}=43.79 \mathrm{E}-13 \\
\sum h_{R}=41.3 \mathrm{E}-13 \\
\sum H_{(x)}^{2}=243.36 \mathrm{E}-26 \\
\left(\sum h_{R}\right)^{2}=203.69 \mathrm{E}-26 \\
\sum H_{(x)} h_{R}=221.985 \mathrm{E}-26
\end{gathered}
$$

Substituting the above values in equation 70 , the correlation coefficient for the restoring equilibrium model is;

$$
r_{R}=0.989879999701
$$

Obviously, a model is usually considered verified when it reproduces historical data within some acceptable level of accuracy and validity, this research has achieved a good match as an evidence of validity of the model as shown in Tables 3 and 4 and also in Figures 7 and 8 . For the critical state condition as shown in Fig. 8, the closest match between the model and observed values is achieved at the $1.8 \mathrm{~m}$ flow distance and a correlation analysis has shown a perfect correlation, $r_{c}$ of 1.00975 . For the equilibrium state condition as shown in Fig.8, the closest agreement has been achieved at $1.8 \mathrm{~m}$ flow distance and the correlation analysis carried out has also shown a perfect correlation, $r_{R}$ of 0.989879999701 . Note, a perfect correlation lies between -1 (perfect negative) and 1 (perfect positive) [22; 23].

\section{CONCLUSION}

The following could be concluded from the present research work;

1. For safe pumping and corresponding yield in the borehole system, inter-granular force between granular particles should equal the seepage force and this is achieved by ensuring that the deduced model expression is used to determine the safe hydraulic head.

2. Finally, as long as the model hydraulic head expression deduced is used under the above conditions, safe pumping can be achieved at any voltage between 150 volts and 240 volts.

\section{ACKNOWLEDGEMENT}

I wish to thank the management of Geodrill Nigeria Limited, Umuahia and Okey Electrical, Aba, Abia State for their assistance in relevant areas to ensure that this work was concluded successfully.

\section{REFERENCES}

[1] Durlofsky, L. J. and Aziz K., "Advanced Technique for Reservior simulation and modelling of nonconventional wells" www.geotechlink.com. Accessed on January 21, 2013 . Final report to Standford University, USA, 2004.

[2] Eduvie, M. O., "Borehole failures and ground water development in Nigeria", A paper presented at a national Seminar held on the occasion of Water Africa Exhibition Lagos, on 15 ${ }^{\text {th }}$ November, 2006.

[3] Munjiza A., The Combined Finite Discrete Element Method. John Wiley and Sons Ltd, England, 2004. 
[4] Sitharam, T. G., Discrete element of modeling of cyclic behaviour of granualr materials, Kluwer Academic Publishers, Geotechnical and Geological Engineering. 2003, 21:297-329.

[5] Cheng, Y. P. Bolton, M. D. \& Nakata, Y., Crushing and Plastic deformation of soils simulation using DEM, Geotechnique 2004, 54 (2),pp.131-141.

[6] Mahabadi, O. K., Lisjak, A., Munjiza, A. and Grasselli, G., New combined Finite-Discrete Element Numerical Code for Geomechanical Applications. Int. J. Geomech ASCE 2012, 12, pp.676-681.

[7] Frederic, V. D., Vincent, R. \& Magnier, S., Advances in DEM applied to Soil, Rock and Concrete Mehcanics, EJGE, 2008, Bouquet 108, pp.1-34.

[8] Fernando, A., Muhlhaus, H. B. \& Herrmann, H.J., "Micromechanical investigation of soil plasticity using a discrete model of polygon particles", Theoretical Applied Mechanics, 2008, Vol. 35, no. 13, pp.11-28.

[9] Bell, N., Yu, Y. \& Mucha, P. J., "Particle based simulation of granualr materials", Eurographics Association, USA, 2005.

[10] Sitharam, T. G. \& Dinesh, S. V., "Numerical Simulation of liquefaction behaviour of granular materials using DEM", Proc. Indian Acad Sci. 2003, 112(3), pp. 479-484.

[11] Sukumaram, B., Das, N \& Ashmawy, A. K., "Modeling granular particle shape using DEM", ${ }^{\text {st }}$ international FLAC/DEM symposium on Numerical modelling, Dubai, 2008.

[12] Lu, H., Anderson M. T., Likos, W. J. \& Mustoe, G. W., "A discrete element model for Kaolinite aggregate formation during sedimentation", International Journal for Numerical and Analytical Methods in Geomechanics, John Wiley and Sons Ltd, USA. 2007.

[13] Fox G. A., Heaven, D. M. Wilson, G. V., Eddy, J. L., Fox, A. K. and Maria L. C., "Numerically predicting seepage gradient forces and erosion sensitivity to soil hydraulic properties" Journal of hydrology, Elsevier, 2010, 389, pp.354-362.

[14] Sivakugan, N., "Permeability and seepage" International Journal of Environment Engineering ASCE, 2005,Vol. 289, pp.1165-1181.

[15] Nwaogazie, I. L., Finite Element Modelling of Engineering Systems with emphasis in Water Resources, Special Publishers, Enugu, 2008. Chaps. 1, $2,3,5$ and 9.

[16] BS 1377. Methods of testing Soils for Civil Engineering purposes. British Standards Institution, Her Majesty's stationery Office, London. 1995.

[17] History of Umuahia Ibeku, Nigeria, www.google.com/historyofumuahiaibeku. Accesed on January 21, 2013

[18] American Society for Testing Materials, ASTM, Annual Book of ASTM Standards, Part 19, Soil and Rock, Philadelphia, PA.

[19] Bardet, J., Experimental Soil Mechanics, Prentice Hall, New Jersey, 1997.

[20] Onyelowe, K.C., "Constant Head Determination of the K-Value of Umudike Aquifer Medium Granular Soil", International Journal of Research in Engineering \& Advanced Technology, Volume 1, Issue 4, Aug-Sept, 2013. ISSN: 2320 - 8791, accessed from www.ijreat.org and www.researchgate.net.

[21] Alaneme, G., "Properties of granular soil and its relevance to civil engineering work", Unpublished Project, 2014. Department of Civil Engineering, Michael Okpara University of Agriculture, Umudike.

[22] Inyama S.C. and Iheagwam, V.A. (1995), Statistics and Probability, a Focus on Hypotheses Testing, Alphabet Nigeria Publishers, Owerri, 1995.

[23] Agunwamba, J.C., Engineering Mathematical Analysis, De-Adroit Innovation, Enugu, 2007. 